\title{
THE STABILITY OF A CURVED, HEATED BOUNDARY LAYER: LINEAR AND NONLINEAR PROBLEMS
}

\author{
C. E. WATSON ${ }^{1}$ and S. R. OTTO ${ }^{2}$
}

(Received 14 May, 2004; revised 23 November, 2004)

\begin{abstract}
We consider the stability of high Reynolds number flow past a heated, curved wall. The influence of both buoyancy and curvature, with the appropriate sense, can render a flow unstable to longitudinal vortices. However, conversely each mechanism can make a flow more stable; as with a stable stratification or a convex curvature. This is partially due to their influence on the basic flow and also due to additional terms in the stability equations. In fact the presence of buoyancy in combination with an appropriate local wall gradient can actually increase the wall shear and these effects can lead to supervelocities and the promotion of a wall jet. This leads to the interesting discovery that the flow can be unstable for both concave and convex curvatures. Furthermore, it is possible to observe sustained vortex growth in stably stratified boundary layers over convexly curved walls. The evolution of the modes is considered in both the linear and nonlinear régimes.
\end{abstract}

\section{Introduction}

The well-known Görtler instability [7] is an important mode within curved boundary layers; the resultant longitudinal counter-rotating vortices have been observed, for example, over the concave surfaces of aeroplane wings [17] and are believed to play an important rôle in the transition of boundary-layer flow to turbulence [11].

Görtler's original work on the subject, combining theory with experimental results, was published in 1940 but it was not until much more recently that the theoretical problem was solved in a consistent manner by Hall [8]. This gave the linear solution to the Görtler equations, including previously erroneously neglected non-parallel terms. In this calculation the spanwise wavelength of the Görtler vortices was taken to be

\footnotetext{
'Quintessa Limited, Dalton House, Newtown Road, Henley-On-Thames, Oxfordshire, RG9 1HG, England.

${ }^{2}$ R\&A Rules Limited, Beach House, Golf Place, St Andrews, KY16 9JA, Scotland; e-mail: steveotto@RandA.org.

(C) Australian Mathematical Society 2005, Serial-fee code 1446-1811/05
} 
significantly smaller than the boundary-layer thickness, resulting in the modes being concentrated in an internal viscous layer which thickens as they grow. It was found that, for a wall of constant radius of curvature, the boundary layer ultimately becomes more stable as the fluid moves downstream.

Following on from this, Hall gave the linear theory for vortices with $O(1)$ wavenumbers [9]. The perturbation equations were shown to be parabolic in the downstream coordinate and thus can be solved using an Euler marching scheme after an initial disturbance form is imposed at the downstream location $x=\bar{x}$. Neutral curves were generated in the $a_{x}-G_{x}$ plane, where $a_{x}$ is the local wavenumber of the vortices (here $a_{x}=a x^{1 / 3}, a$ being the actual vortex wavenumber) and $\mathrm{G}_{x}$ is the local Görtler number (here $\mathrm{G}_{x}=\mathrm{G} \chi(x) x^{5 / 3}$ ): these quantities reflect the fact that the layer spreads as it evolves downstream. The Görtler number, $\mathrm{G}$, is a non-dimensional entity which is the ratio of centrifugal to viscous forces and the function $\chi(x)$ contains all the $x$-dependence of the curvature. The Görtler number is positive for concave curvatures and negative for convex curvatures. These neutral curves were found to be non-unique in the sense that the growth rate of an imposed disturbance depends upon both how it is introduced into the flow and the location of the introduction; hence the necessity for the inclusion of the non-parallel terms. However, the right-hand branch of the neutral curve is unique (for a particular set of physical parameters) and an asymptotic solution may be calculated for it. It was discovered that a vortex of fixed wavelength is locally unstable for a finite distance along the boundary layer with a wall of constant curvature. Hence as the fluid moves downstream, any vortex flow will eventually decay to zero; at least in the absence of nonlinear effects. But if the curvature of the wall increases at a sufficient rate then stability is never reached and the flow remains unstable for an infinite distance downstream, although in reality nonlinear terms come into play to substantially modify the flow.

The nonlinear solution to the full Görtler equations (rather than in the limit of a large vortex wavenumber, as given by, for example, Hall and Lakin [13]) is attainable only by numerical means [10], but of course provides much better agreement with experimental results. This is demonstrated by Lee and Liu [16] who compare their results with the experimental findings of Swearingen and Blackwelder [24]; good qualitative agreement is obtained. The addition of nonlinear mode interaction enables distortion of the flow which is not possible with the linear theory. Thus the characteristic mushroomshaped streamwise velocity profiles are predicted (see for example Benmalek and Saric [1]), which develop as the counter-rotating vortices pull fluid away from the wall region.

Denier and Hall [5] extended the linear theory of Denier, Hall and Seddougui [6] by examining the nonlinear development of the most unstable Görtler mode in the boundary layer. It was found that this mode resides within a viscous layer near the wall; but perhaps the most relevant finding here is that eventually a singularity is 
encountered as the solution is marched downstream and the numerical method breaks down. This is caused by reverse flow within the boundary layer, which violates the parabolic nature of the governing equations.

The Görtler instability is centrifugally driven. A similar thermally-driven instability also exists within the boundary layer [26]. As in the case of work on the Görtler instability, parallel-flow theories were the norm until Hall and Morris [14] emphasised that non-parallel effects are important and must be considered. The compressible Navier-Stokes equations were simplified by way of the Boussinesq approximation, which essentially removes all dependence on density variation except where it is coupled to the gravitational acceleration in the buoyancy force. This results in governing equations which are virtually the same as those for an incompressible fluid but with the addition of an energy equation and an extra term, $\mathrm{Gr} T$, in the $y$-momentum equation. The non-dimensional number $\mathrm{Gr}$ is known as the Grashof number and is the ratio of buoyancy to viscous forces, whilst $T$ is the dimensionless temperature. Solutions of these equations were found numerically at $O(1)$ values of the Grashof number; and the triggering of instabilities by wall roughness, non-uniform wall heating and free-stream disturbances was investigated. This parameter régime is poignant to atmospheric problems, Scorer [21]. The case of $\mathrm{Gr} \gg 1$ was studied and the most unstable mode found, which occurs at a high wavenumber. At low wavenumbers the vortex instability is shown to take on the characteristics of a Tollmien-Schlichting wave (this phenomenon is not restricted purely to Grashof modes).

Centrifugal and thermal effects may act constructively or destructively within the boundary layer to affect the susceptibility of the flow to counter-rotating, longitudinal vortex instabilities which often still bear the name of Görtler but perhaps more accurately should be called Görtler-Grashof vortices. The competing aspects of buoyancy and curvature have been studied by Stott and Denier [23], again using the Boussinesq approximation. In the linear régime, taking $\mathrm{Gr} \gg 1$ and assuming $S$ is negligible (where $S$ represents the level of buoyancy coupling), it was shown that the effects of curvature are first felt when the Görtler number is of an order comparable to the Grashof number. For a positive or concave curvature a negative temperature gradient (that is, unstable stratification) will increase the growth rates of already unstable modes. If the curvature is convex, the flow is unstable with growth rates decreasing as the Görtler number becomes more negative (that is, as the curvature gets more and more convex). Therefore eventually the stabilising influence of the convex curvature will overcome the destabilising effect of buoyancy. No unstable vortex modes could be found for stably-stratified flows. If $S$ is considered non-negligible and buoyancy coupling is introduced, the effects of curvature are felt in the basic flow when the local slope of the body (and hence the local Görtler number) is of order one. In this case, the curvature is introduced through the buoyancy or curvature induced acceleration in the basic flow rather than through the perturbation equations. For a given amount of 
buoyancy coupling, it was discovered that a sufficiently large convex curvature would stabilise any mode. In the nonlinear régime the combined limit of large $\mathrm{Gr}$ and $\mathrm{G}$ was taken. It was found that the effect of buoyancy is to enhance the nonlinear vortex motion and allow the vortices to exist in parameter ranges where the centrifugal effects alone are not sufficient to support them. The boundary layer may also be accelerated by the nonlinear vortex state, increasing the maximum value of the streamwise velocity.

This paper aims to extend the findings of Stott and Denier [23] into régimes where the vortex wavenumber is of $O(1)$. Initially the linear problem will be considered and the effects of introducing buoyancy coupling discussed. This will be continued in the fully nonlinear régime, using the methods of Denier and Hall [5].

\section{Formulation}

We consider the boundary layer which arises in the flow over a slowly curving, heated wall with a radius of curvature given by $1 / \kappa$ and a temperature $T_{0}$. In the free stream away from the wall, the fluid has a velocity of $U_{\infty}$ in the streamwise direction and a temperature of $T_{\infty}$. This system is governed by the continuity, Navier-Stokes and energy equations and the Boussinesq approximation [22] is made, eliminating full compressibility but retaining thermal effects. The usual non-dimensionalisations are made, resulting in four dimensionless parameters. The first of these is the Reynolds number, given by $\operatorname{Re}=U_{\infty} L / v$. Here $L$ is a typical length scale and $v$ is the kinematic viscosity of the fluid. We consider problems which are characterised by large Reynolds numbers. The Grashof and Görtler numbers also appear after non-dimensionalisation and are given by the expressions

$$
\mathrm{Gr}=\frac{g \alpha L^{3} \Delta T}{\nu^{2}} \mathrm{Re}^{-3 / 2} \quad \text { and } \quad \mathrm{G}=2 L \kappa \mathrm{Re}^{1 / 2}
$$

respectively, where $g$ is the acceleration due to gravity and $\alpha$ is the coefficient of thermal expansion. The quantity $\Delta T=T_{0}-T_{\infty}$ is the temperature difference between the wall and the free stream at infinity. Thus a positive Grashof number indicates heating from below (an unstable stratification) whilst a negative Grashof number indicates cooling from below (a stable stratification). The final dimensionless quantity is the Prandtl number, defined as $\operatorname{Pr}=\rho_{m} c_{p} v / k$. Here $\rho_{m}$ is the mean density of the fluid, $c_{p}$ is the specific heat capacity at constant pressure and $k$ is the thermal conductivity. The Prandtl number will be regarded as an order one constant throughout; in fact in the main it will be taken to be unity since its variation is found to have minimal effect here.

After rescaling on the boundary-layer thickness (equal to $\mathrm{Re}^{-1 / 2}$ ) in both the normal, $y$, and spanwise, $z$, directions, the flow is split into the basic component (denoted 
by barred variables) and a perturbation (denoted by variables with tildes). Thus

$$
\begin{aligned}
(u, v, w, T) & =\left(\bar{u}, \operatorname{Re}^{-1 / 2} \bar{v}, 0, \bar{T}\right)+\left(\tilde{u}, \operatorname{Re}^{-1 / 2} \tilde{v}, \operatorname{Re}^{-1 / 2} \tilde{w}, \tilde{T}\right), \\
p & =\bar{p}_{0}(x)+\operatorname{Re}^{-1 / 2} \bar{p}_{1}(x)+\mathrm{S} \bar{p}(x, y)+\operatorname{Re}^{-1} \tilde{p} .
\end{aligned}
$$

In these expressions: basic flow quantities (unless otherwise stated) are functions of $x$ and $y$; and those with tildes, that is, perturbations, are functions of $x, y$ and $z$. The flows in the streamwise $x$, normal $y$ and spanwise $z$ directions are $u, v$ and $w$ respectively. Note that the pressure is expanded in a slightly different manner to the other variables; this is following the method introduced by Hall [12]. The quantity $S$ which appears here is known as the buoyancy parameter and is equivalent to $\mathrm{Gr} / \mathrm{Re}$. It enables one to adjust the amount of buoyancy present within the system.

2.1. The basic flow The basic flow satisfies the set of equations

$$
\begin{aligned}
\bar{u}_{x}+\bar{v}_{y} & =0, \\
\bar{u} \bar{u}_{x}+\bar{v} \bar{u}_{y} & =u_{e} u_{e x}-\mathrm{S} \bar{p}_{x}+\frac{1}{2} \mathrm{SG} \psi(x) \bar{p}_{y}+\bar{u}_{y y}, \\
\bar{p}_{y} & =\bar{T}, \\
\bar{u} \bar{T}_{x}+\bar{v} \bar{T}_{y} & =\frac{1}{\operatorname{Pr}} \bar{T}_{y y},
\end{aligned}
$$

where $u_{e} u_{e x}=-\bar{p}_{0 x}$, using the Bernoulli equation. Physically $u_{e}(x)$ represents the value that the streamwise velocity takes as $y \rightarrow \infty$, that is, the velocity parallel to and removed from the plate.

Equation (2.1b) contains two buoyancy-induced acceleration terms, the second of which is only present when the boundary layer is curved. Here the function $\psi(x)$ is equivalent to the gradient of the wall, whereas $\chi(x)$ is the wall's curvature. In addition to these two terms, Equations 2.1 differ from the equivalent equations for the incompressible case by the fact that $(2.1 \mathrm{c})$ introduces a buoyancy-induced normal pressure gradient.

To solve basic-flow equations (2.1), similarity solutions of the Falkner-Skan type [3] will be used. A similarity variable is introduced as $\eta=y x^{-1 / 3}$ and the flow variables take the form $\bar{u}=x^{1 / 3} f^{\prime}(\eta), \bar{v}=x^{-1 / 3}\left[\eta f^{\prime}(\eta)-2 f(\eta)\right] / 3, \bar{p}=x^{2 / 3} q(\eta)$ and $\bar{T}=x^{1 / 3} g(\eta)$. These satisfy the coupled set of nonlinear ordinary differential equations

$$
\begin{aligned}
f^{\prime \prime \prime}-\frac{1}{3}\left(f^{\prime}\right)^{2}+\frac{2}{3} f f^{\prime \prime}+\frac{1}{3} & =\frac{2}{3} S q-\frac{1}{3} S \eta q^{\prime}-\frac{1}{2} S G^{\mathrm{loc}} q^{\prime}, \\
q^{\prime} & =g, \\
\frac{1}{\operatorname{Pr}} g^{\prime \prime}+\frac{2}{3} f g^{\prime}-\frac{1}{3} f^{\prime} g & =0,
\end{aligned}
$$


where a prime denotes a derivative with respect to $\eta$. These equations must be solved subject to the boundary conditions

$$
\begin{aligned}
& f, f^{\prime}=0 \text { and } \quad g=1 \text { on } \eta=0 \text {, } \\
& f^{\prime} \rightarrow 1 \text { and } g, q \rightarrow 0 \text { as } \eta \rightarrow \infty \text {. }
\end{aligned}
$$

If $S$ is set to zero, (2.2a) reduces to the Hiemenz equation [3], rather than the Blasius equation [2] as the external velocity, $u_{e}$, has been chosen to vary as $x^{1 / 3}$ (see Itoh [15] for a discussion of the more general Falkner-Skan similarity solution). Finally, the term, $\mathrm{G} \psi(x)$, which is representative at the wall's gradient must be taken as $\mathrm{G}^{\mathrm{loc}} x^{-2 / 3}$ to permit the similarity solution to exist, where $\mathrm{G}^{\mathrm{loc}}$ is an order one constant known as the local Görtler number since it describes only the local gradient rather than the overall curvature. To avoid confusion later when the term "local Görtler number" will have a different meaning, $G^{\text {loc }}$ will be referred to by symbol only from now on.

Equations (2.2) are then solved numerically using a Runge-Kutta routine coupled with a Secant shooting method. As described by Stott and Denier [23], it is possible to accelerate or decelerate the streamwise component of the velocity from the solution obtained when the buoyancy parameter is zero by varying the value of $\mathrm{G}^{\text {loc }}$, which we recall is representative of the wall's gradient. Here we consider a stably stratified layer, such that $S<0$ and typical solutions are shown in Figure 1.

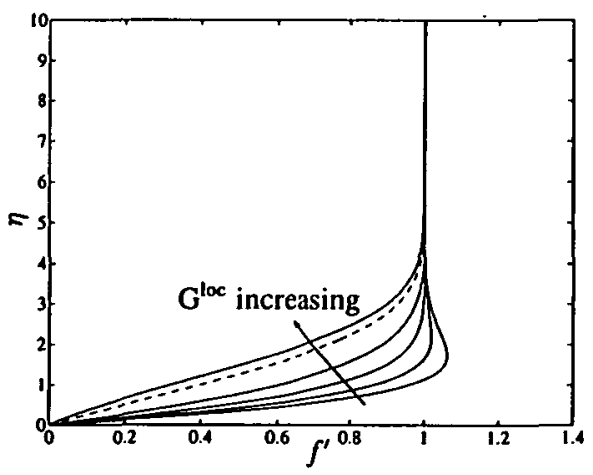

FIGURE 1. The similarity solution for the basic-flow streamwise velocity, $\vec{u}=X^{1 / 3} f^{\prime}$; calculated for $G^{\text {loc }}=-8,-6,-4,-2,0$ (dashed line) and $1 / 2$ with $S=-1 / 2$ (corresponding to a stable temperature stratification). Here decreasing the value of $\mathrm{G}^{\text {loc }}$ causes the streamwise velocity to eventually overshoot the free-stream value.

It should be noted that, as stated by Stott and Denier, for increasingly negative values of $\mathrm{G}^{\mathrm{loc}}$ a new inflection point emerges from $\eta=0$ and ultimately the similarity solution will break down as the boundary layer becomes detached from the wall. We note that as the flow is accelerated in the neighbourhood of the wall, that is, as $\mathrm{G}^{\text {loc }}$ decreases, we encounter supervelocities. This flow necessarily contains an inflection 
point and hence we realise that the flow will be prone to inviscid travelling waves (as it will for $G^{\text {loc }}>0$ ).

2.2. The disturbance equations We now discuss the equations governing the perturbations. After making some simple re-arrangements, Hall [9], the equations which govern the disturbance are found to be

$$
\begin{aligned}
& \mathscr{L} \tilde{u}-\bar{u}_{x} \tilde{u}-\bar{u}_{y} \tilde{v}=Q_{1}, \\
& \mathscr{L}\left(\frac{\partial^{2}}{\partial y^{2}}+\frac{\partial^{2}}{\partial z^{2}}\right) \tilde{v}-\bar{v}_{y} \tilde{v}_{y y}+\bar{u}_{x y} \tilde{v}_{y}+\bar{u}_{y y} \tilde{v}_{x}+\bar{u}_{x y y} \tilde{v}+\bar{v}_{x} \tilde{u}_{y y}+2 \bar{u}_{x} \tilde{u}_{x y} \\
& +2 \bar{u}_{x y} \tilde{u}_{x}+\left(-\left(\bar{v}_{x}+\mathrm{G} \chi \bar{u}\right) \frac{\partial^{2}}{\partial z^{2}}+\bar{u}_{x x y}\right) \tilde{u}+\mathrm{Gr} \tilde{T}_{z z} \\
& =-Q_{1 x y}+Q_{2 z z}-Q_{3 y z} \quad \text { and } \\
& \mathscr{L}_{\tau} \tilde{T}-\bar{T}_{x} \tilde{u}-\bar{T}_{y} \tilde{v}=Q_{4},
\end{aligned}
$$

where the two partial differential operators are given by

$$
\begin{aligned}
& \mathscr{L} \equiv \frac{\partial^{2}}{\partial y^{2}}+\frac{\partial^{2}}{\partial z^{2}}-\bar{u} \frac{\partial}{\partial x}-\bar{v} \frac{\partial}{\partial y} \quad \text { and } \\
& \mathscr{L}_{\mathrm{r}} \equiv \frac{1}{\operatorname{Pr}}\left(\frac{\partial^{2}}{\partial y^{2}}+\frac{\partial^{2}}{\partial z^{2}}\right)-\bar{u} \frac{\partial}{\partial x}-\bar{v} \frac{\partial}{\partial y}
\end{aligned}
$$

here a subscript denotes a derivative with respect to that variable. The four nonlinear terms on the right-hand sides of (2.3) are

$$
\begin{array}{ll}
Q_{1}=\tilde{u} \tilde{u}_{x}+\tilde{v} \tilde{u}_{y}+\tilde{w} \tilde{u}_{z}, & Q_{2}=\tilde{u} \tilde{v}_{x}+\tilde{v} \tilde{v}_{y}+\tilde{w} \tilde{v}_{z}+(1 / 2) \mathrm{G} \chi \tilde{u}^{2}, \\
Q_{3}=\tilde{u} \tilde{w}_{x}+\tilde{v} \tilde{w}_{y}+\tilde{w} \tilde{w}_{z}, & Q_{4}=\tilde{u} \tilde{T}_{x}+\tilde{v} \tilde{T}_{y}+\tilde{w} \tilde{T}_{z} .
\end{array}
$$

When studying the linear problem, these terms will simply be set to zero. At this stage we have retained virtually the full equations, however the rescaling of the normal and spanwise coordinates has led to the demotion of the streamwise diffusion and streamwise pressure gradient. This implies that the equations are parabolic in $x$ and are thus amenable to solution by a marching technique.

Equations (2.3) (a modified form of the nonlinear Görtler equations due to the presence of the buoyancy term) must be solved subject to the condition that all disturbances are required to die away at the edge of the boundary layer, that is, $\tilde{u}$, $\tilde{v}, \tilde{T}, \tilde{v}_{y} \rightarrow$ as $y \rightarrow \infty$ (the condition on the derivative of $\tilde{v}$ comes from continuity combined with the fact that $\tilde{w} \rightarrow$ as $y \rightarrow \infty$ ). In addition, the no-slip and noflux conditions must be applied at the wall as well as ensuring that the temperature perturbation is zero here. We use this isothermal condition, since we are taking the 
wall to be maintained at a prescribed.temperature. However, in our experience the use of an adiabatic condition does not have much effect on the dominant physical mechanisms, although it does modify the numerical values of the critical quantities. Thus we have $\tilde{u}, \tilde{v}, \tilde{T}, \tilde{v}_{y}$ are all zero at $y=0$. We now discuss the numerical methods employed to solve the disturbance equations (2.3).

\section{Numerical methods}

The disturbance equations (2.3) will be solved using a second-order Crank-Nicolson scheme in the downstream direction coupled with a centred five-point fourth-order finite difference method in the normal direction. This method is suitable for both the linear and the nonlinear problems, with small modifications required for the latter, and will follow that used by, for example: Hall [9], Denier and Hall [5] and Cole, Otto and Watson [4]. Appropriate checks were made to ensure the fidelity of the numerical solutions, for instance on grid sensitivity. In addition we looked at the effect of the grid resolution on the breakdown of the nonlinear code and this was found to have a minor effect. In essence the results we shall detail are not dependent on the accuracy of the schemes being used.

3.1. The linear problem To obtain the linear disturbance equations, the four nonlinear terms $Q_{1}, Q_{2}, Q_{3}$ and $Q_{4}$ are set to zero in (2.3). Effectively we consider the perturbation to be proportional to an infinitesimally small quantity and hence we can legitimately neglect these nonlinear terms. Using experimental knowledge the perturbation quantities are taken to be periodic in the spanwise coordinate since we are looking for vortex instabilities and so we write

$$
\tilde{u}(x, y, z) \mapsto u(X, \eta) \mathrm{e}^{i a z}, \quad \tilde{v}(x, y, z) \mapsto v(X, \eta) \mathrm{e}^{i a z}, \quad \tilde{T}(x, y, z) \mapsto T(X, \eta) \mathrm{e}^{i a z} .
$$

Here the transformation $(x, y) \mapsto(X, \eta)$ has also been made, where $X=x$ and $\eta=y x^{-1 / 3}$ as before. The use of the similarity variable not only simplifies use of the basic-flow results but also allows for the downstream spreading that the boundary layer experiences.

The system of equations (2.3), once transformed, must be solved subject to the containing boundary conditions $u=v=T=v_{\eta}=0$ on $\eta=0$ and $u, v, T, v_{\eta} \rightarrow 0$ as $\eta \rightarrow \infty$. In order to obtain a numerical solution, the normal domain must be truncated at a finite value and this occurs at $\eta=40$. This value has been demonstrated to be sufficiently large so the vortices are unaffected by the presence of the false boundary.

As mentioned previously the system (2.3) is parabolic in the downstream coordinate, $X$, and so a forward marching scheme may be used to solve the system. An 
initial condition is required and this is chosen to be

$$
u=\eta^{6} \mathrm{e}^{-\eta^{2}}, \quad v=T=0,
$$

as used by Hall [9]. The choice of this form of disturbance and its point of inception, together with other choices (for instance the point at which the nonlinear calculations are started) has a bearing on the numerical results attained herein. However, this does not alter the central findings of this paper relating to the physical mechanisms which support these instabilities. The dominant effects of the initial form of the disturbance are concentrated in the initial stages, which are seen in the transients and these in turn influence the left-hand branch of the neutral curve. In contrast the right-hand branch of the neutral curve is largely unaffected by these choices and it is this which gives the most information concerning the fate of a particular situation. The evolution of this disturbance must be tracked downstream and this is achieved by measuring an energy at each step in $X$, using the formula

$$
\mathscr{E}^{(n)}(X)=\int_{0}^{\infty}\left[u^{(n)}(X, \eta)^{2}+T^{(n)}(X, \eta)^{2}\right] \mathrm{d} \eta .
$$

The superscript $(n)$ is used in readiness for the nonlinear problem but it is superfluous here. From this energy a spatial growth rate is then derived, namely

$$
\sigma^{(n)}(X)=\frac{1}{\mathscr{E}^{(n)}} \frac{\mathrm{d} \mathscr{E}^{(n)}}{\mathrm{d} X}+\frac{1}{3 X} ;
$$

the particular form of this expression comes from the fact that the integration in (3.2) is performed using the similarity variable $\eta$. Unsurprisingly, it is this growth rate which will be monitored to determine the growth of the disturbance, at least in the linear problem. If it is negative, the disturbance is decaying and conversely if it is positive, the disturbance is growing. Intermediate points where the growth rate is necessarily zero are known as neutral points and are used to plot neutral curves in the local Görtler number-local wavenumber plane. These two quantities allow for the spreading of the mixing layer as it progresses downstream and are defined by $\mathrm{G}_{X}=\mathrm{G} \chi(X) X^{5 / 3}$ and $a_{X}=a X^{1 / 3}$. The curvature function, $\chi(X)$, is chosen to be equal to $(X / \bar{X})^{1 / 3}$ : the functional form of the curvature is chosen to promote sustained downstream growth. Here $\bar{X}$ is the value of the streamwise coordinate where the marching procedure is started and hence corresponds to the imposition of the disturbance. In the calculation included herein it is universally taken to be $\bar{X}=20$; the effect of the variation of this quantity is described in Hall [9] for the Blasius boundary later. Before proceeding we add the caveat that the form of the disturbance (3.1) has an effect on the upcoming results. In order to replicate and predict the fate of disturbances within a given experiment one would need to initiate calculations with appropriate starting conditions. Alternatively one can effect a receptivity calculation, Watson et al. [25]. 
3.2. The nonlinear problem We now describe the solution of the nonlinear problem, the underlying mechanics of which are similar to the linear problem. The presence of the nonlinear terms $Q_{1}, Q_{2}, Q_{3}$ and $Q_{4}$ will generate the higher harmonics and it is necessary for us to extend the expansion of the flow quantities to include these. Hence we now consider

$$
\begin{aligned}
& \tilde{u}(x, y, z)=\sum_{s=-\infty}^{\infty} u^{(s)}(X, \eta) \mathrm{e}^{i s a z}, \quad \tilde{v}(x, y, z)=\sum_{s=-\infty}^{\infty} v^{(s)}(X, \eta) \mathrm{e}^{i s a z}, \\
& \tilde{w}(x, y, z)=\sum_{\substack{s=-\infty \\
s \neq 0}}^{\infty} w^{(s)}(X, \eta) \mathrm{e}^{i s a z}, \quad \tilde{T}(x, y, z)=\sum_{s=-\infty}^{\infty} T^{(s)}(X, \eta) \mathrm{e}^{i s a z},
\end{aligned}
$$

where $a$ is the vortex wavenumber, so the period of the vortex is $2 \pi / a$. We note that the linear analysis corresponds to consideration of the quantities with superscripts of plus one in isolation. Obviously the limits of the sums must be replaced by finite values for computational reasons and therefore they will be truncated at plus and minus $N$. Provided $N$ is sufficiently large this will introduce a negligible error since the modes will get smaller and smaller as $|s|$ increases - here $N=8$ is found to be adequate. Extensive studies have been performed to ensure this is true. The $s=0$ mode is dubbed the 'basic-flow correction' since, unlike the other modes but similar to the basic flow, it is not periodic in $z$. There is no $w^{(0)}$ term in the sum since, as stated by Hall [10], it is well known that the nonlinear interactions do not generate a mean flow in the spanwise direction.

The spanwise velocity, $w^{(s)}$, appears in the nonlinear terms and so a further equation in addition to (2.3) is required. This is the continuity equation, given by

$$
u_{X}^{(s)}-\frac{\eta}{3 X} u_{\eta}^{(s)}+\frac{1}{X^{1 / 3}} v_{\eta}^{(s)}+i a s w^{(s)}=0 .
$$

The boundary conditions are of course the same as the linear conditions, that is, $u^{(s)}=v^{(s)}=w^{(s)}=T^{(s)}=v_{\eta}^{(s)}=0$ on $\eta=0$ and $u^{(s)}, v^{(s)}, w^{(s)}, T^{(s)}, v_{\eta}^{(s)} \rightarrow$ as $\eta \rightarrow \infty(s \neq 0)$. As mentioned above, the case $s=0$ corresponds to the basic-flow correction and since there is no spanwise velocity correction, the number of equations to solve may be reduced from four to three. The three chosen are naturally (2.3a), (2.3c) and (3.4). The boundary conditions for this mode are also slightly different; the conditions on the streamwise velocity and the temperature remain the same, but those on the normal velocity are required to match that of the basic flow. Thus it is simply enforced that there is no normal flux through the plate, that is, $v^{(0)}(0)=0$.

The method of solution of the governing equations also remains the same as in the linear case, that is, a centred five-point finite difference scheme is used in the normal direction and a Crank-Nicolson marching scheme in the downstream direction. The linear solution is used as an initial condition for the nonlinear problem; that is, the 
linear problem is evolved from $X=20$ to $X=40$, at which point the nonlinear terms are activated and the nonlinear problem is solved. The linear solution has just a single mode whilst the nonlinear has $2 N+1$, therefore the linear solution is used to seed just two of the nonlinear modes, the $s= \pm 1$ modes, in the manner

$$
\begin{aligned}
\left(u^{(1)}, v^{(1)}, w^{(1)}, T^{(1)}\right) & =\frac{\Delta_{u}}{\sqrt{\mathscr{E}^{(1)}}}\left(u^{\text {linear }}, v^{\text {linear }}, w^{\text {linear }}, T^{\text {linear }}\right) \\
\left(u_{\bar{n}}^{(-1)}, v_{\bar{n}}^{(-1)}, w_{\bar{n}}^{(-1)}, T_{\bar{n}}^{(-1)}\right) & =\frac{\Delta_{u}}{\sqrt{\mathscr{E}^{(1)}}}\left(u^{\text {linear }}, v^{\text {linear }}, w^{\text {linear }}, T^{\text {linear }}\right)^{*}, \\
\left(u^{(s)}, v^{(s)}, w^{(s)}, T^{(s)}\right) & =(0,0,0,0), \quad(s \neq \pm 1),
\end{aligned}
$$

where a star denotes a complex conjugate and $\mathscr{E}^{(1)}$ is the mode energy as defined by (3.2). The parameter $\Delta_{u}$ controls the strength of the nonlinearity in the initial stages of growth. The effects of changing this value have been discussed by Cole, Otto and Watson [4] -it was found that increasing $\Delta_{u}$ has the effect of reducing the growth rates of the vortices, at least for the fundamental, although it does accelerate the breakdown of the calculation. The amplitude of the linear modes for $20<X<40$ are rescaled accordingly to match with the nonlinear calculations. This is essentially just a rescaling so that the reference amplitude for the calculations is taken at $X=40$ using a mode for which some of the transients associated with an artificial starting condition have been allowed to abate (at least somewhat). In the nonlinear problem each mode will have an energy associated with it and so (3.2) will be used for each $-N \leqslant s \leqslant N$. In the main the energy of the modes will be used in the description of the system rather than growth rates or neutral curves. This is due to the fact that these latter quantities are less poignant within a nonlinear context, especially when discussing higher harmonics.

The method chosen to initiate the nonlinear problem produces an even distribution across the Fourier modes, that is, $u^{(-s)}=\left(u^{(s)}\right)^{*}$, and so on for the other flow variables. This fact may be exploited to reduce computational effort since it is known that $u^{(s)}$, $v^{(s)}$ and $T^{(s)}$ are real whilst $w^{(s)}$ is purely imaginary-hence only half of the Fourier modes need to actually be calculated. At each streamwise location it is necessary to iterate to a solution of the nonlinear system; this is accomplished using Newton's method [4].

\section{Linear results}

We begin by solving the linear system, using the method described in Section 3.1. The two cases of a zero and a non-zero buoyancy parameter will be considered separately. The former case is relatively well understood, or at least when either curvature or buoyancy is present-the boundary layer experiencing the combined 


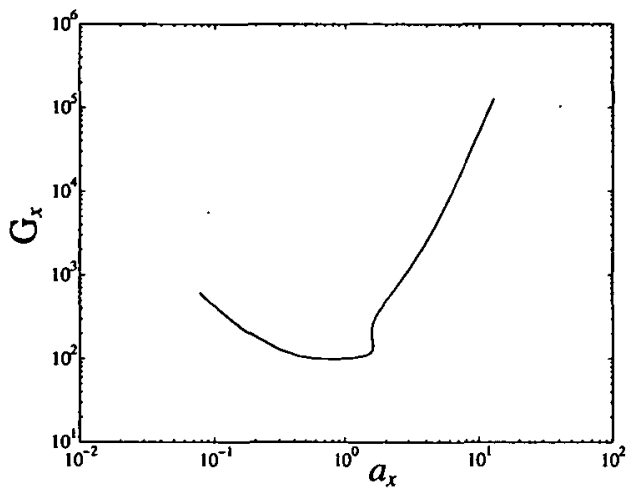

FIGURE 2. Neutral curve for a stably-stratified basic flow over a wall with curvature $G=0.5$ and a zero buoyancy parameter. Even with this fairly large degree of concave curvature, the curve is already folded.

effects of curvature and buoyancy has not been studied in depth (with the exception of the inviscid theory of Stott and Denier [23]) but the effects are easily surmised from knowledge of both the individual problems $[9,11,14]$ and the equivalent mixing layer problem [18-20]. The buoyancy-coupled boundary layer has received little attention in the literature and thus will be the main focus of attention here.

In the remainder of this paper we shall be referring to "stably" and "unstably" stratified basic-flow temperature profiles. Recall that the non-dimensional temperatures at the wall and in the free stream are unity and zero respectively. We add the remark that the non-dimensional temperature of zero is merely a scaled value and does not represent an extreme of temperature. The stratification of the flow is then controlled via the Grashof number, which is a function of $\Delta T=T_{0}-T_{\infty}$, the dimensional temperature difference between the wall and the free stream. Thus a positive Grashof number represents heating from the wall, an unstable stratification, whilst a negative Grashof number represents cooling from the wall -a stable temperature stratification. We shall use just two values of the Grashof number, plus and minus one, to represent a stable and an unstable stratification respectively.

4.1. No buoyancy coupling $(S=0)$ As already stated, when buoyancy coupling is removed the basic-flow equations (2.2) are solved by Hiemenz flow.

For a Görtler number of 0.5 and a stably stratified layer, growth is sustained for a range of wavenumbers as shown by the neutral curve (Figure 2), although the curve already shows signs that the boundary layer is subject to the same transient growth as described by Watson, Otto and Jackson [25] for the equivalent mixinglayer problem. As with that situation, it can be shown that the modified TaylorGoldstein equation which governs the inviscid stability of the boundary layer has purely imaginary eigenvalues, representing oscillatory motion in the downstream 
coordinate.

In the $G=0.2$ case, growth is sustained, leading to a neutral curve similar to that shown in Figure 2 but with a more pronounced folding (not shown due to the similarity to Figure 2). However, in the $G=0.05$ case there is no growth at all and the disturbance decays throughout the downstream section shown. Once again, this situation mirrors that of the curved, heated mixing layer. It has been shown [25], via the consideration of the inviscid stability boundaries, that all mixing layers with a stable stratification and a concave centreline curvature will experience a period of transient growth before a certain downstream position is reached, at which point growth will become sustained. This occurs only when the centreline curvature is a function of the downstream coordinate, $X$. Exactly the same phenomenon occurs in the boundary layer. The inviscid stability boundaries for the boundary layer may not be expressed in such a succinct form as they could for the mixing layer; however, one may nevertheless ascertain that the critical Görtler number (the amount of wall curvature required to destabilise the system given a temperature stratification) $\sim X^{-1 / 3}$ for a stable stratification. This is, of course, completely dependent upon the choice of curvature function, $\chi(X)$.

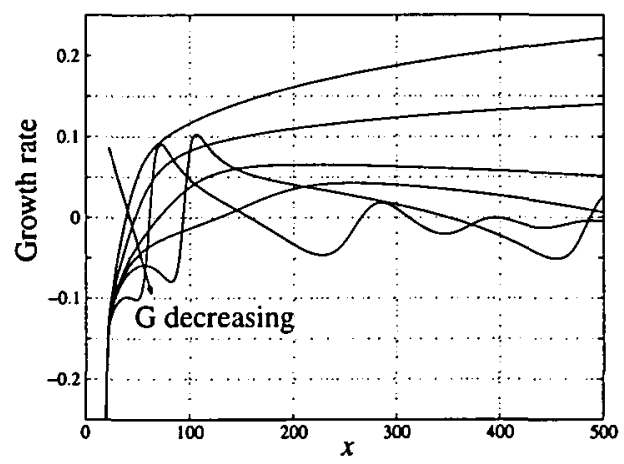

FIGURE 3. Typical growth rates for unstably-stratified basic flows. The vortex wavenumber was 0.05 and $S=0$. We show curves for Görtler numbers of $0.2,0.05,-0.05,-0.075,-0.1$ and -0.2 .

In Figure 3 growth rate curves are presented for a range of Görtler numbers within an unstably-stratified basic flow (achieved by setting the Grashof number to plus one). When the Görtler number becomes negative, the growth rate initially increases but reaches a maximum and then proceeds to become negative, indicating decay. This is exactly the same behaviour as seen in the mixing layer [25] when the basic flow was unstably stratified; analysis of the inviscid stability boundaries in that case showed that, at negative values of the Görtler number, the disturbance would initially grow but at some point downstream it would experience decay (or transient growth) and the period of growth would never be regained. At positive values of the Görtler number, growth 
would be sustained at all points downstream. For an unstably-stratified boundary layer, it is found that the critical Görtler number $\sim-X^{-1 / 3}$; which is a direct consequence of the choice of the curvature. This in turn is chosen to permit a similarity reduction of the equations. If the curvature exceeds this value then we would expect the instabilities to have greater growth and vice versa for a lesser curvature. This would be associated with a smaller or larger critical Görtler number respectively.

4.2. Buoyancy coupling $(S \neq 0$ ) The case of a non-zero buoyancy parameter will now be considered, allowing strong buoyancy coupling within the basic flow equations. Stott and Denier [23] examined the consequences of this upon the inviscid stability of the boundary layer and it was found that growth rates could be increased or decreased depending upon the local gradient of the wall. In this section it will be seen whether this result extends out of the inviscid régime or not.

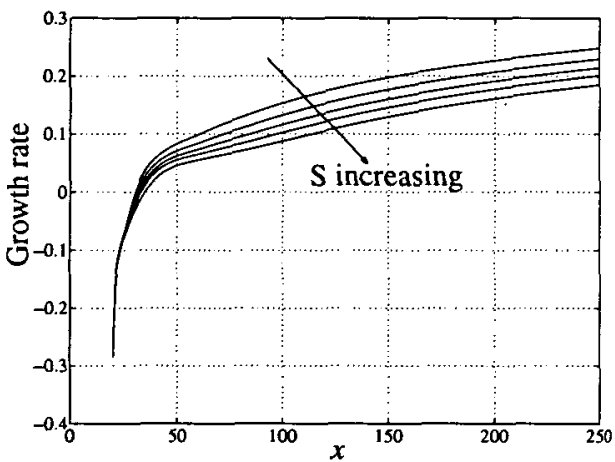

(a) $\mathrm{G}^{\text {loc }}=1 ; \mathrm{S}=-0.4,-0.3,-0.2,-0.1$ and 0 .

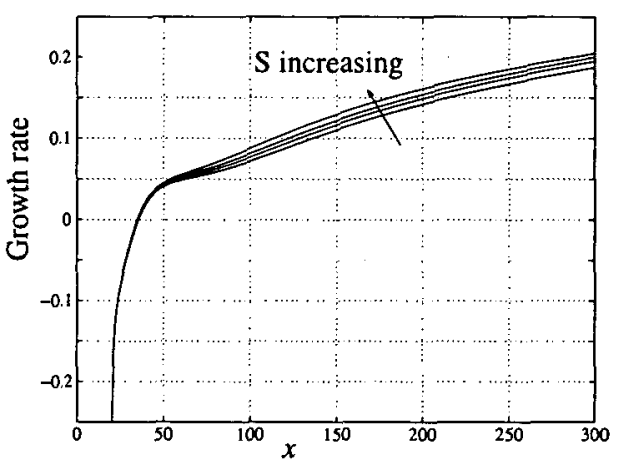

(b) $\mathrm{G}^{\mathrm{loc}}=-3 ; \mathrm{S}=-0.3,-0.2,-0.1$ and 0 .

FIGURE 4. Growth rate curves for a variety of values of the buoyancy parameter. The basic-flow temperature profile was stably stratified, the Görtler number was 0.5 and the vortex wavenumber was 0.05. In the second figure, since the basic flow is relatively decelerated in the streamwise direction, increasing the buoyancy parameter increases the growth rate, refer to Figure 1.

In Figure 4 (a) growth rate curves are plotted for a Görtler number of 0.5 and a stably-stratified basic flow. The vortex wavenumber was, as usual, 0.05 and $\mathrm{G}^{\text {loc }}$ was unity. The buoyancy parameter, $S$, is increased from a negative value to zero (note that the sign of $S$ is equal to the sign of the Grashof number) - as it increases, the growth rate decreases. This is as found by Stott and Denier [23] when studying the inviscid limit; the accelerated boundary-layer basic flow was found to yield much smaller growth rates than the decelerated boundary layer.

It would be expected, therefore, that if $G^{\text {loc }}$ is chosen such that the flow near the wall is decelerated rather than accelerated, increasing the buoyancy parameter would have the opposite effect - the growth rate would be increased. This can be seen in 
Figure 4 (b), which again shows growth rates for various values of the buoyancy parameter but this time $G^{\text {loc }}=-3$. The greater the degree of deceleration, the larger the growth rate of the disturbance.

In Figure 5 the consequences of varying $\mathrm{G}^{\text {loc }}$ are shown, with the buoyancy parameter fixed at $-1 / 2$. The effect of decelerating the streamwise flow near the wall is clearly seen once again, as the growth rate is largest when $\mathrm{G}^{\text {loc }}$ is positive.

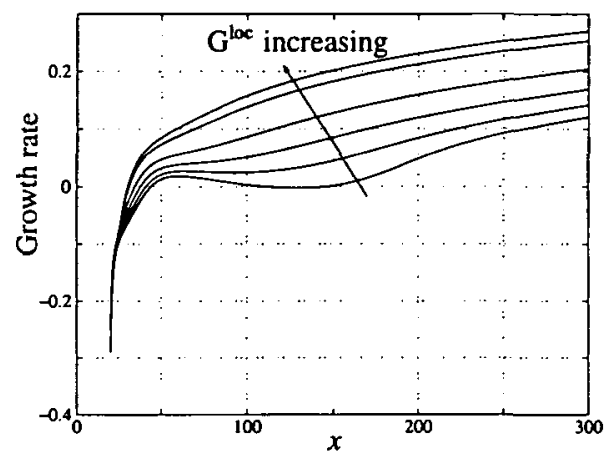

(a) $a=0.05$

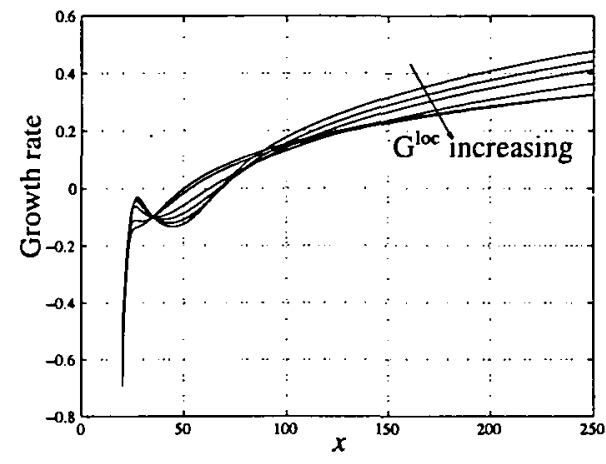

(b) $a=0.6$

FIGURE 5. Growth rate curves for the stably-stratified boundary layer with the buoyancy parameter fixed at $-1 / 2$ but $\mathrm{G}^{\mathrm{loc}}$ varying between -8 and $1 / 2$ (specifically $\mathrm{G}^{\mathrm{loc}}=-8,-6,-4,-2,0,1 / 2$ ) and the Görtler number was 0.5 .

Figure 5 (a) also shows that transient growth appears when $-8 \gtrsim G^{\text {loc }}$, indicating that the stability boundaries are changed by the inclusion of buoyancy coupling at least for these initial conditions. This is hardly surprising since the inviscid stability boundaries are dependent upon the basic flow, which is clearly modified by allowing buoyancy coupling.

Neutral curves and their right-hand branches are of course also affected by the inclusion of buoyancy coupling, as changes in the basic flow will naturally alter the stability characteristics of the flow. Figure 6 shows a number of neutral curves for a stably-stratified basic flow, each calculated with a different value of $\mathrm{G}^{\text {loc }}$ as shown in the legend.

As $\mathrm{G}^{\mathrm{loc}}$ is decreased, the right-hand branch moves further to the right. However, the kink also grows corresponding to our earlier observation that transient growth occurs when $\mathrm{G}^{\text {loc }}$ is decreased. In fact, by the time $\mathrm{G}^{\text {loc }}$ has been decreased to -8 , the kink is so large that it extends across the whole range of local wavenumbers used to produce this figure, effectively splitting the neutral curve into two parts.

The relative positions of the right-hand branches shown in Figure 6 is somewhat surprising given the results of Figure 5 (a), which indicated that the more negative the value of $G^{\text {loc }}$, the smaller the growth rate will be. If a similar figure is plotted but the 


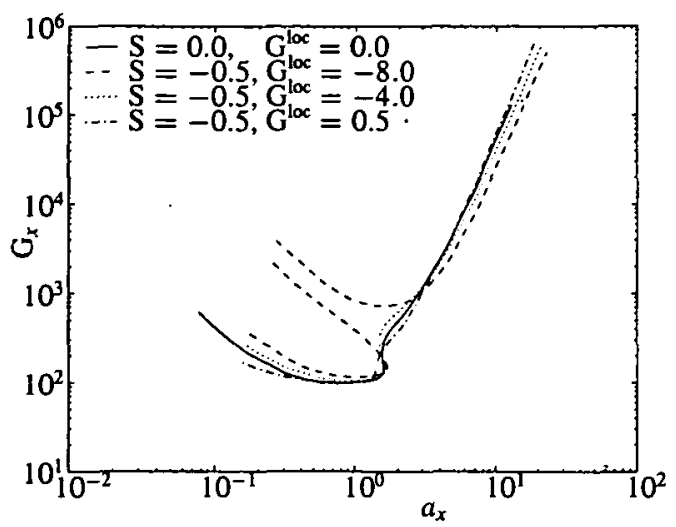

FIGURE 6. Neutral curves calculated for a variety of values of $G^{\text {loc }}$ and a stably-stratified basic flow. The Görtler number was 0.5 .

vortex wavenumber is increased to 0.6 , it is clear that the situation is reversed; see Figure 5 (b). This time, the acceleration in the boundary-layer basic flow increases rather than decreases the growth rate once $X$ is sufficiently large. This scenario is rather easier to justify physically, in that there is more energy available to the disturbance in the boundary layer if the basic flow is accelerated beyond the free-stream value. Why this situation should be reversed if the vortex wavenumber is reduced is unclear-it appears that in this régime, the extra energy is directed into spatial oscillations rather than sustained downstream growth. This is linked to the stability boundaries, which are changed according to the value of $\mathrm{G}^{\mathrm{loc}}$. Some of the variation can be attributed to a horizontal shifting of the neutral curve.

The basic-flow temperature profile is now changed so that it is unstably stratified; the corresponding growth rates are shown by Figures 7 (a) and 7 (b). The former of these two figures shows the growth rates for various values of $G^{\text {loc }}$, at a vortex wavenumber of 0.05 and a Görtler number of 0.5 . In this case, in contrast to the stably-stratified basic flow, increasing the value of $G^{\text {loc }}$ decreases the growth rate. Figure 7 (b) shows the growth rates when the wavenumber is unity; increasing $G^{\text {loc }}$ now increases the growth rate. Figure 8 shows a number of neutral curves, each calculated for a different value of $G^{\text {loc }}$, as shown in the legend. Also included is the neutral curve calculated when no buoyancy coupling is present. In contrast, the greater the value of $G^{\text {loc }}$, the more prone to longitudinal vortex instabilities the system is and the further to the right the right-hand branch lies.

We now proceed to discuss the analytical derivation of the location of the right-hand branch. 


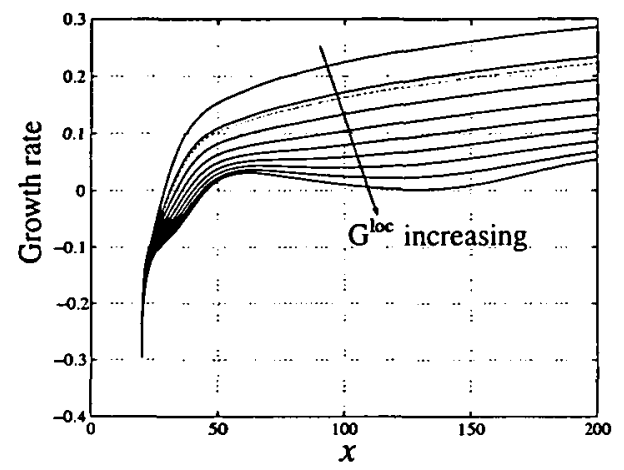

(a) $a=0.05$

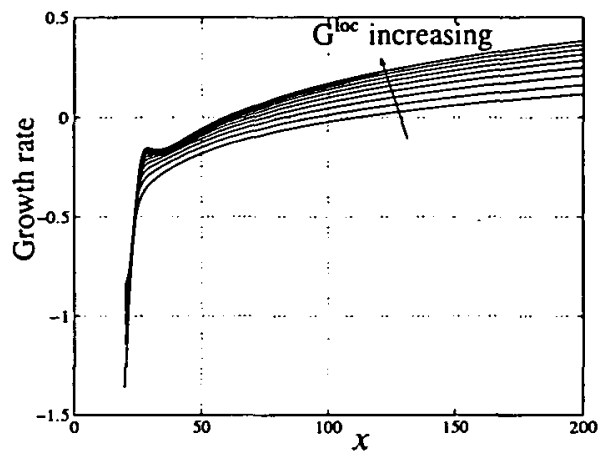

(b) $a=1$

FIGURE 7. Growth rate curves for integer values of $G^{\text {loc }}$ varying between -3 and 5 . The buoyancy parameter was unity and the Görtler number 0.5 with an unstably-stratified basic-flow temperature profile. The dotted line in (a) shows the growth rate for the same parameters but with $S=0$.

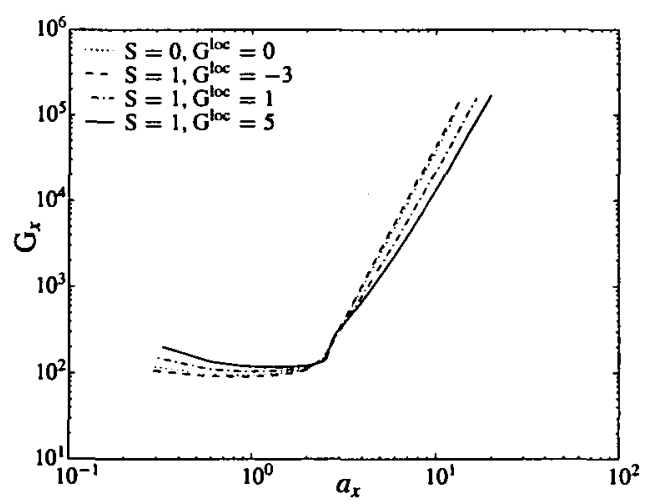

FIGURE 8. Neutral curves for a variety of values of $G^{\text {loc }}$, with an unstable temperature stratification. The right-hand branches of the curves occur moving from left to right as: $G^{\text {loc }}=-3,0,1$ and 5 .

4.3. The right-hand branch asymptote The results in the previous section indicate that the right-hand branch is dependent upon the values of the buoyancy parameter and $G^{\text {loc }}$. Using an analysis identical to that detailed in Watson, Otto and Jackson [25] (with scalings appropriate to the Hiemenz boundary layer rather than the mixing layer) one may derive an expression for the right-hand branch asymptote in the limit of a large vortex wavenumber. We note in this high wavenumber limit the vortices become concentrated within a narrow layer. We expand both the perturbation and basic flow quantities within this layer, and we find that conditions for the existence of the instabilities are

$$
1-\mathrm{G}_{0} \bar{u}_{0} \bar{u}_{1}+\operatorname{Pr} \bar{T}_{1}=0 \text { and }-\mathrm{G}_{0}\left(\bar{u}_{0} \bar{u}_{2}+\bar{u}_{1}^{2}\right)+\operatorname{Pr} \bar{T}_{2}=0,
$$




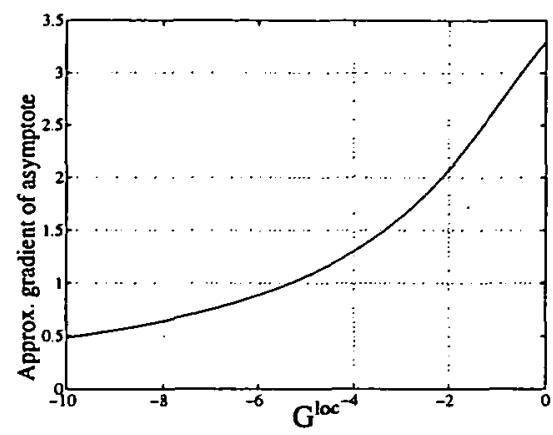

(a) The upper branch.

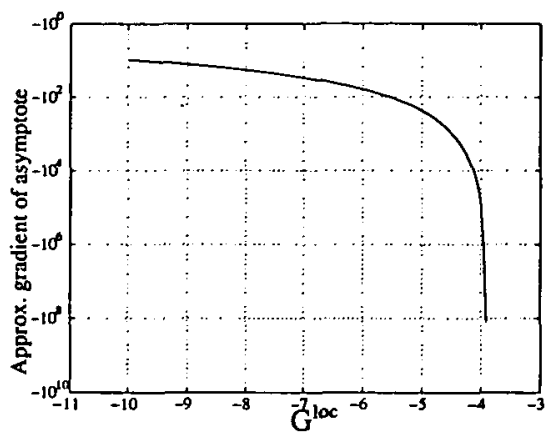

(b) The lower branch.

FIGURE 9. The variation of the gradient of the right-hand branch asymptote with the parameter $\mathrm{G}^{\text {loc }}$ with $\mathrm{S}=-1 / 2$. The upper branch is depicted in (a), whilst (b) shows the lower branch (note the logarithmic scale on the vertical axis).

where the quantities $\bar{T}_{1}, \bar{u}_{0}, \bar{u}_{1}$ and $\bar{u}_{2}$ are terms in the expansions of the basic flow as defined by Watson, Otto and Jackson [25]. These define both the value of $G_{0}$ (for neutrality) and the normal location of the modes. The leading term in the asymptote is

$$
\mathrm{G}_{X}=\frac{\left(1+\operatorname{Pr} \bar{T}_{1}\right)}{\bar{u}_{0} \bar{u}_{1}} a_{X}^{4}+\cdots
$$

Subsequent terms in the expression for the asymptote are naturally smaller than this one and will be neglected here.

Using (4.1) we are able to calculate the approximate gradient of the right-hand branch asymptote (4.2) for a given basic flow (that is, the gradient when the asymptote is plotted in the log-log plane). This is shown in Figure 9 (a) for the buoyancy-coupled boundary layer with varying values of $G^{\text {loc }}$ (the buoyancy parameter was fixed at $-1 / 2$ ). Clearly the larger the value of $G^{\text {loc }}$, the further to the right the asymptote lies - the steeper its gradient is -indicating greater instability. This agrees entirely with the numerical results of Section 4.2.

However, a rather surprising result also comes to light with this analysis - when the buoyancy parameter is non-zero, two different solutions exist of the above compatibility conditions and thus it appears that two different right-hand branch asymptotes exist. One of these always has a positive gradient, the other a negative gradient and this latter case is shown in Figure 9 (b) for various values of $\mathrm{G}^{\mathrm{loc}}$. The differing signs mean that each asymptote is only appropriate for one sign of the curvature.

We note, as mentioned above, the compatibility relations (4.1) also yield information concerning the location of the vortices within the high wavenumber limit. We note that for $S<0$ and $G^{\text {loc }}<0$ the jet weakens and appears to tend out into the freestream. It is relatively straightforward to show that the vortex associated with the lower branch (that is, negative Görtler number or convex curvatures) will reside 


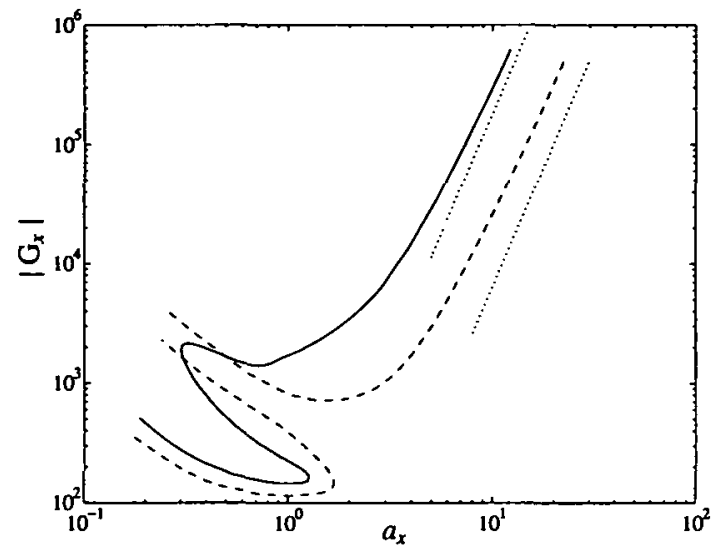

FIGURE 10. Neutral curves for the stably-stratified boundary layer, with Görtler numbers of -0.5 (representing a convex wall curvature-solid curve) and 0.5 (representing a concave wall curvaturedashed curve). In both cases, the basic-flow velocity profile was accelerated beyond the free-stream value in the streamwise direction since $S=-0.5$ and $G^{\text {loc }}=-8$. The dotted lines represent the right-hand branch asymptotes, calculated analytically.

above the jet and the upper branch mode will reside below it. This, coupled with the multiplicative factor of $\bar{u}_{1}$ (the local shear at the vortex location), implies that the Görtler number associated with neutrality for the convex modes will become very large and hence these modes will only be expected to be observed for highly curved situations. However, we note that as $G^{\text {loc }}$ decreases and the jet strengthens we would expect the modes to be observed in moderately curved situations.

Consequently as $\mathrm{G}^{\mathrm{loc}}$ becomes increasingly negative, the gradient of this lower branch tends to minus infinity very rapidly thus rendering it obsolete, just as it is in the mixing layer, the Blasius and Hiemenz boundary layers. Yet it is certainly a very important factor when the basic flow is accelerated in the streamwise direction. This can be seen in Figure 10, which shows neutral curves for a stably-stratified boundary layer with both a convex curvature characterised by a Görtler number of -0.5 (dotted line) and a concave curvature characterised by a Görtler number of 0.5 (continuous line). Note that the absolute value of the local Görtler number is plotted so that both curves can be shown in the same figure; thus both right-hand branches appear to have positive gradients.

In the standard Hiemenz boundary layer the former situation (stable stratification, convex curvature) would be completely stable to longitudinal vortex instabilities. However this is not the case in the buoyancy-coupled boundary layer with $S=-1 / 2$, $\mathrm{G}^{\mathrm{loc}}=-8$ as can be seen from this neutral curve. The asymptotes, calculated analytically, are shown as a dotted line in each case and it is clear that each neutral curve tends to a separate asymptote. In the case where the wall has a concave curvature 
with a Görtler number of 0.5 , it becomes difficult to extend the numerical results much further along the right-hand branch, hence the apparent lack of correlation with the asymptote. As one moves further along the right-hand branch, the step size in the downstream coordinate must be made smaller and smaller to avoid the occurrence of a numerical instability. It becomes infeasible to reduce this parameter much further with the computational means and time available to us. However, enough of the right-hand branch has been calculated to clearly show that it does not tend to the same asymptote as the right-hand branch of the neutral curve corresponding to $\mathrm{G}=-0.5$.

\subsection{A note on the inviscid stability boundaries in the case of non-zero buoyancy} coupling $(\mathbf{S} \neq \mathbf{0})$ The inviscid stability boundaries have been mentioned frequently in previous sections, as they predict the transient growth which has been observed. They may be expressed succinctly in the mixing-layer problem; in the boundary-layer problem (with $S=0$ ) they are not so neatly written but they are calculable and their influence is clearly seen. However, upon the introduction of buoyancy coupling, the inviscid stability boundaries no longer exist in the same form. It is observed by Stott and Denier [23] that the modified Taylor-Goldstein disturbance equation takes the form

$$
v_{0 y y}-\frac{1}{\beta_{0} \bar{u}}\left[a^{2}\left(\beta_{0} \bar{u}+\frac{\operatorname{sgn}(\mathrm{Gr}) \bar{T}_{y}}{\beta_{0} \bar{u}}\right)+\beta_{0} \bar{u}_{y y}\right] v_{0}=0,
$$

when $S \neq 0$ (first derived by Hall and Morris [14]), where $v_{0}$ is the re-scaled (on the Grashof number) normal disturbance velocity. This equation lacks the curvature term $a^{2} \mathrm{G}_{0} \chi \bar{u} \bar{u}_{y} v_{0} / \beta_{0}^{2} \bar{u}^{2}$ which appears in the corresponding disturbance equation when buoyancy coupling is not present [23]. The difference arises because the curvature term is an $O(1)$ entity when buoyancy coupling is present and is thus negligible when compared to the other terms. The effects of the underlying wall curvature are not evident explicitly in the inviscid problem, but are implicit within the basic flow. With no curvature term present in the modified Taylor-Goldstein equation, the analysis of Otto, Stott and Denier [18] cannot be performed and thus stability boundaries may not be derived in the conventional way. It is possible that a consideration of the smaller terms will yield the stability boundaries but this calculation is rather involved and is beyond the scope of this paper.

Indeed, the search for the stability boundaries is complicated further by the fact that sustained growth of the disturbance has been observed at values of the Görtler number which are less than zero (representing a convex wall curvature), even when the basic flow has a stable temperature stratification. However, transient growth is also seen in some cases (in particular when the vortex wavenumber is small-see for example Figure $5(a)$ ) and so one can surmise that instability does not persist for all values of $\mathrm{G} \chi(X)$ and hence stability boundaries do exist in some form, however they 
are unlikely to be accessible by simple analytical means. We now proceed to discuss the nonlinear evolution of these modes, since after sustained streamwise growth it is expected that nonlinearity will play a rôle.

\section{Nonlinear results}

The full nonlinear problem will now be solved, using the method outlined in Section 3.2. As described there, the method is very similar to that used to solve the linear problem in the previous section. However, there are now an infinite number of nonlinear modes which should be considered (albeit that this is truncated to a finite number, as also described in Section 3.2, for computational purposes). As mentioned previously, traditionally, the growth of these nonlinear modes is shown not by growth rate and neutral curves but by plotting the energy of each mode as it progresses downstream.

5.1. No buoyancy coupling $(\mathbf{S}=\mathbf{0}$ ) We begin by returning to the problem with no buoyancy coupling present. As before, in all cases the Prandtl number will be taken as unity. The equivalent incompressible problem has been previously studied by Hall [10], Lee and Liu [16], Denier and Hall [5] and Benmalek and Saric [1] (amongst others). These all use the Blasius basic flow which does not vary with $X$ far from the wall, unlike the Hiemenz basic flow which shall be used here; thus the results are not only a precursor to those which follow for the buoyancy-coupled boundary layer but are also new in their own right.

The energy of the first nine nonlinear modes in the stably-stratified boundary layer, calculated for a concave wall curvature characterised by a Görtler number of 0.5 , are shown in Figure 11 (a). The vortex wavenumber was $0.05, \Delta_{u}=0.05$ and the step size used in the downstream coordinate was 0.2 . Figure 11 (b) shows an enlarged view of the mode energies close to the breakdown of the code; the basic-flow correction does not outgrow the fundamental mode as is usually expected $[4,5]$. In fact, the second mode soon becomes larger than the basic-flow correction itself and it is this mode which becomes almost as large as the fundamental mode.

As experienced in similar situations $[4,5]$, the numerical code is subject to failure at a certain point downstream. With these parameters, this occurs at approximately $X=140$. It is found that, unlike the mixing layer, the boundary layer is not overly affected by the value of $\Delta X$, the step size in the downstream coordinate. Although the breakdown point does vary, the range is limited. The fact that the breakdown point moves further downstream as the step size is increased is probably caused by the code "stepping over" the true breakdown value, also noted by Denier and Hall [5]. It is only the breakdown point which is affected to any appreciable degree by the value of $\Delta X$; the energies of the modes undergo only minute changes and thus a step size of 0.2 will 


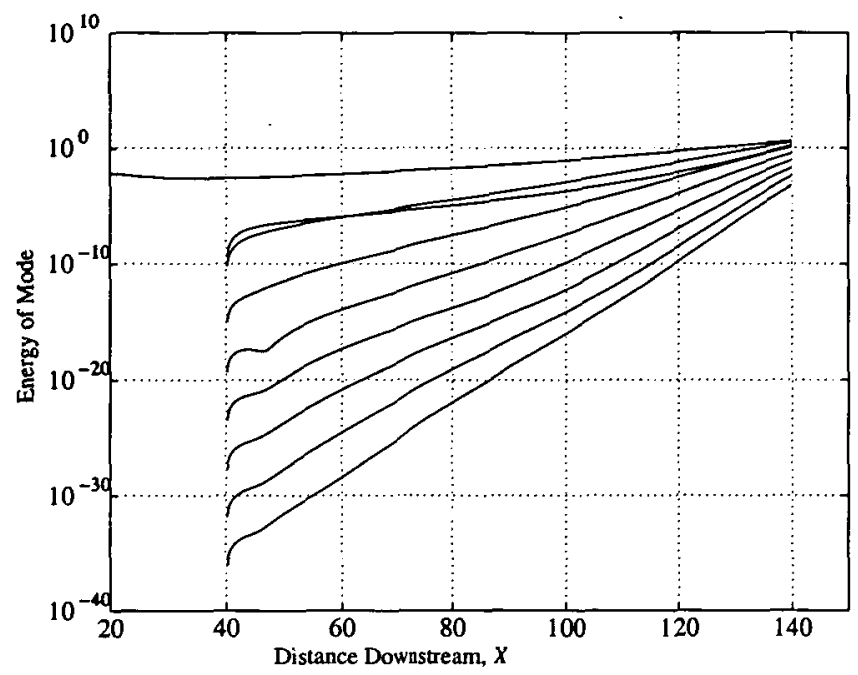

(a) Mode energies

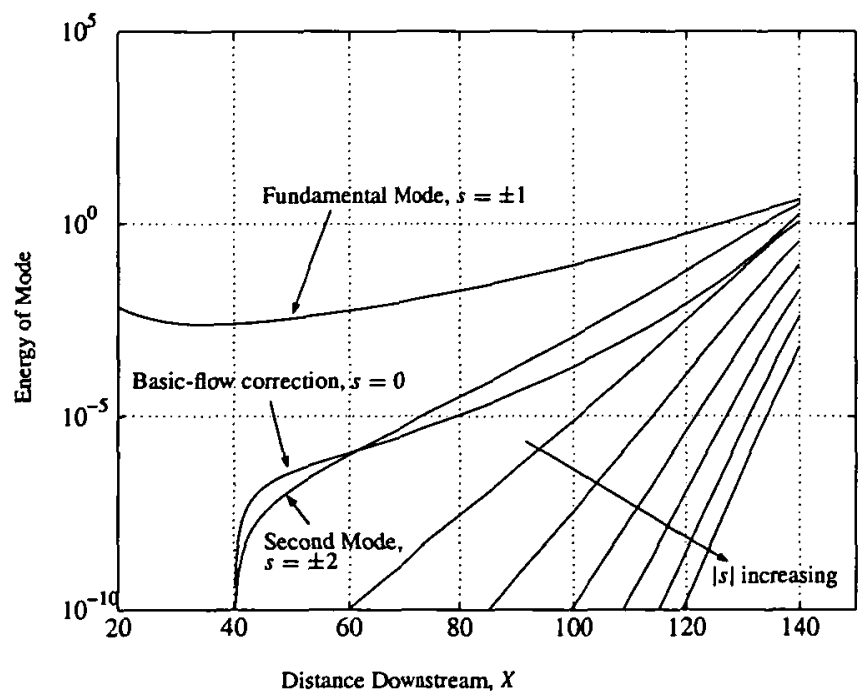

(b) Expansion of (a)

FIGURE 11. Energy of the first nine nonlinear modes in the stably-stratified boundary layer, calculated for $\mathbf{G}=0.5$ and $S=0$. The vortex wavenumber was 0.05 and the step size in the downstream coordinate was 0.2 . In (b) we show a enlargement of Figure 11 (a), showing how the second mode $(s= \pm 2)$ outgrows the basic-flow correction and almost becomes as large as the fundamental mode prior to code breakdown. 
be implemented from now on. Although this does allow the code to bypass the true breakdown point slightly, it greatly reduces the time needed to compute the solution.

In Figure 12 contour plots of the streamwise component of the vorticity and the total temperature (this is the dimensionless temperature, so although it appears that the wall is hotter than the fluid far away from it this is not the case in real terms) are shown once the vortices have developed for the above configuration (stable stratification, $\mathrm{G}=\mathbf{0 . 5}$ ).

Two pairs of vortices are present. In this case the lower pair or the wall vortices are the strongest; the upper pair are weaker and become quite distorted in the latter stages of growth before breakdown. The action of the counter-rotating vortex pairs acts to pull fluid up away from the wall, as seen by the pillars formed in the temperature profile. Within this column of fluid a negative velocity may be observed; this region of reverse flow undoubtably is the root of code breakdown as it was in the Blasius boundary layer as studied by Denier and Hall [5]. The horizontal axis of the contour plots covers one vortex wavelength (equivalent to $2 \pi / a$ ); the fluid pulled away from the wall remains very concentrated in a region in the middle of this wavelength and the scale used makes it seem very thin. In fact zooming in on the pillar shows that it has the characteristic mushroom shape, as seen previously in the incompressible boundary layer $[1,10,16]$.

The energies of the nonlinear modes for the same configuration but with a larger vortex wavenumber $(a=0.6)$ are shown in Figure 13. The first notable difference between these energies and those shown in Figure 11 is that the basic-flow correction is now no longer outgrown by the second mode and indeed it eventually becomes larger than the fundamental mode. Breakdown is also delayed slightly by increasing the vortex wavenumber; however, with the notable exception of the basic-flow correction, the energies of the modes are decreased (particularly those of the smaller modes).

In Figure 14 contour plots of the total streamwise vorticity and temperature are given for the larger vortex wavenumber.

It is clear that now the upper vortex pair is the strongest; the lower pair are very close to the wall indeed and are much weaker. As the disturbance grows downstream, the upper vortices sink towards the wall and their centres move closer together. As before, the effect of the vortices is to pull fluid away from the wall up towards the free stream, but this time the fluid spreads out right across the region shown (again one vortex wavelength) rather than being concentrated in the centre. In the head of the column there is a jet of almost stationary, super-heated fluid. Once again this trend towards flow reversal is the root of code breakdown.

Growth is severely curtailed if the Görtler number is reduced to 0.2 whilst the basicflow temperature profile remains stably-stratified; there is a long period of decay, as shown by Figure 15, followed eventually by growth.

The transition from decay to growth is quite rapid although it is found to be smooth 


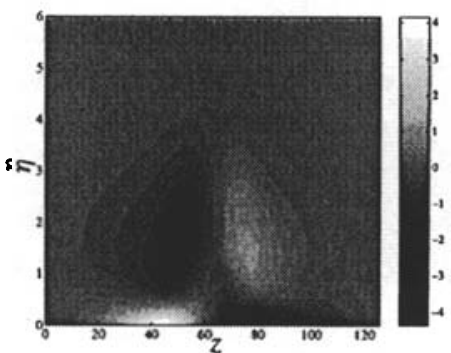

(a) Streamwise vorticity

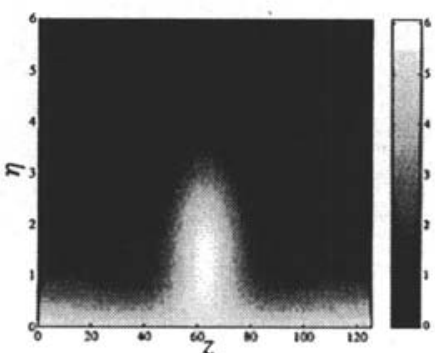

(b) Temperature

FIGURE 12. The total streamwise vorticity and temperature in the stably-stratified boundary layer, calculated for $\mathrm{G}=0.5$ with $a=0.05$ and $\mathrm{S}=0$, shown a reasonable distance downstream. The wall vortices are the most dominant at this value of the vortex wavenumber.

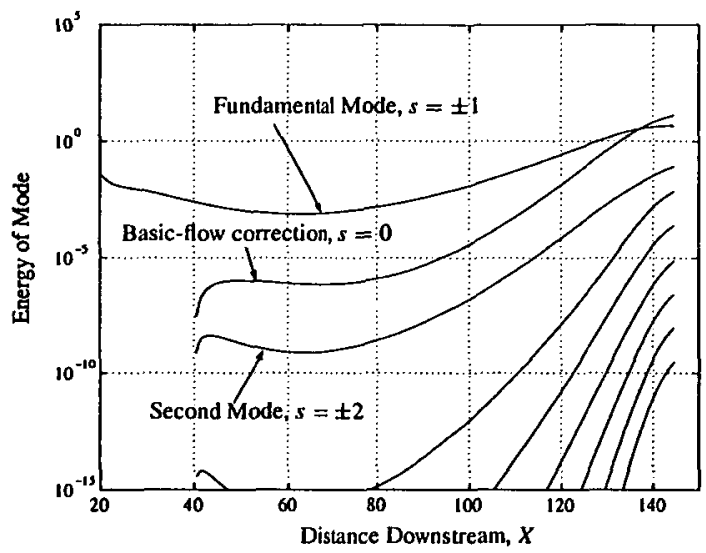

FIGURE 13. Energy of the first nine nonlinear modes in the stably-stratified boundary layer, calculated for $\mathrm{G}=0.5$, the vortex wavenumber was 0.6 and $S=0$. The graph shows that the larger vortex wavenumber has enabled the basic-flow correction to outgrow the second mode and eventually also the fundamental mode.

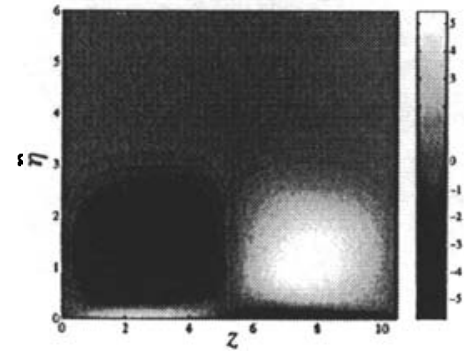

(a) Streamwise vorticity

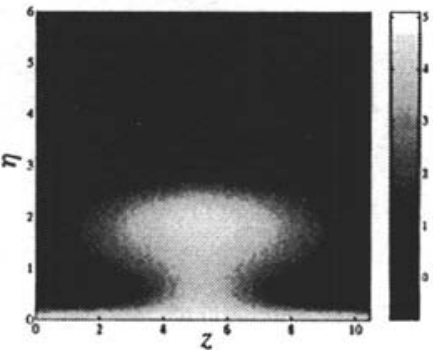

(b) Temperature

FIGURE 14. The total streamwise vorticity and temperature in the stably-stratified boundary layer, calculated for $G=0.5$ with $a=0.6$ and $S=0$. In this case the upper vortex pair are stronger than the wall vortices. 


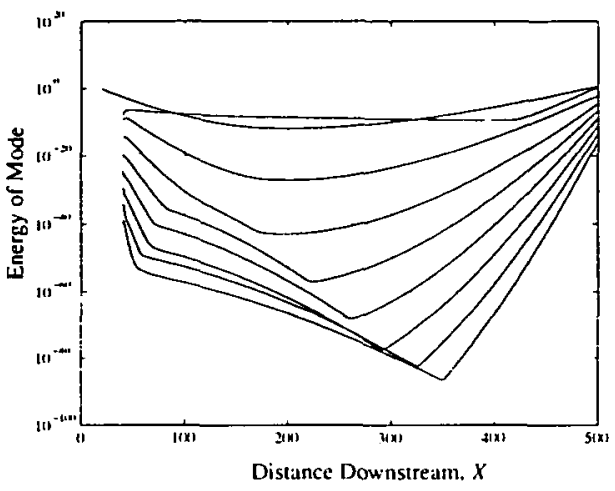

FIGURE 15. Energy of the first nine nonlinear modes in the stably-stratified boundary layer, calculated for $\mathrm{G}=0.2$. The vortex wavenumber was 0.6 and $\mathrm{S}=0$. Code breakdown is not achieved before $X=500$.

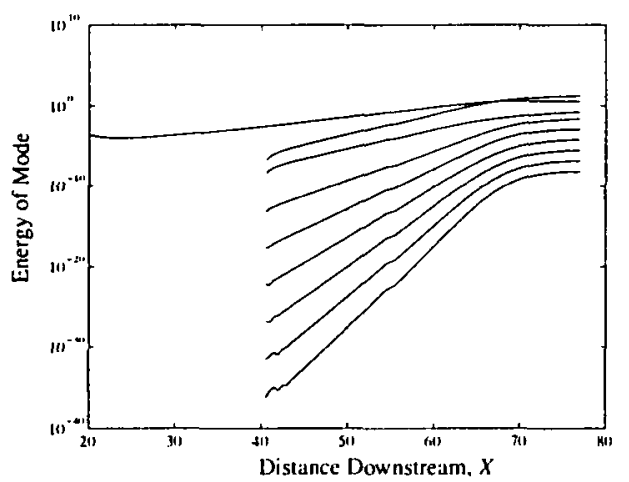

FIGURE 16. Energy of the first nine nonlinear modes in the unstably-stratified boundary layer, calculated for $G=0.5$. The vortex wavenumber was 0.6 and $S=0$. The larger wavenumber means that now the basic-flow correction remains larger than the second mode throughout and indeed outgrows the fundamental mode shortly before code breakdown.

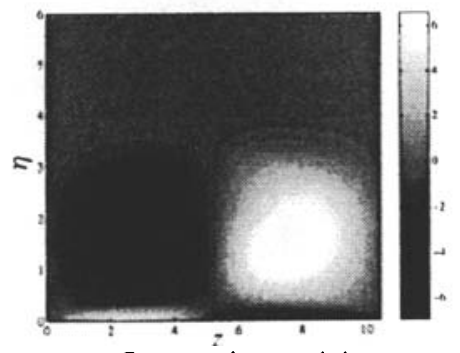

(a) Streamwise vorticity

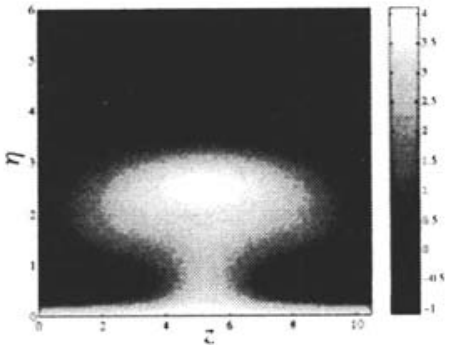

(b) Temperature

FIGURE 17. The total streamwise vorticity and temperature in the unstably-stratified boundary layer, calculated for $\mathrm{G}=0.5$ with $a=0.6$ and $S=0$. 
by examining the apparent cusps of Figure 15 in greater detail. Once again the transition is predicted by the inviscid stability boundaries; the curvature is concave but the temperature profile is stably-stratified, hence there must be a period of transient growth (or decay) followed by sustained growth. The system was integrated to $X=500$ but no breakdown point was encountered in this period as growth is so slow.

We now consider an unstably-stratified basic-flow profile, with a wall curvature characterised by a Görtler number of 0.5 . Energies of the first nine nonlinear modes for a vortex wavenumber of 0.6 are shown in Figure 16. Comparison to the corresponding stably-stratified case shown in Figure 13 shows that the added instability of the unstable stratification causes the energies to increase more rapidly and the code to break down sooner. This can also be seen in the contour plots of the streamwise vorticity and total temperature (Figure 17) which, although similar to their stably-stratified counterparts (Figure 14), show that the vortices not only pull fluid away from the wall more quickly but also extend further towards the free stream. Figures 18 (a) and 18 (b) show the effects of reducing the Görtler number to 0.2 and 0.05 respectively, maintaining the unstable stratification of the basic flow. The vortex wavenumber is once again 0.6. In both cases the code carries on for quite a while downstream after the basic-flow correction has outgrown the fundamental mode, before breaking down. However, after this occurrence, growth slows down considerably and the energies increase only gradually before breakdown is reached. In the latter case, when the Görtler number is 0.05 , the code carries on for so long that the upwellings of fluid begin to merge with each other. Therefore in Figure 19 (which shows contour plots of the streamwise vorticity and total temperature) four wavelengths are shown rather than just one to illustrate this point.

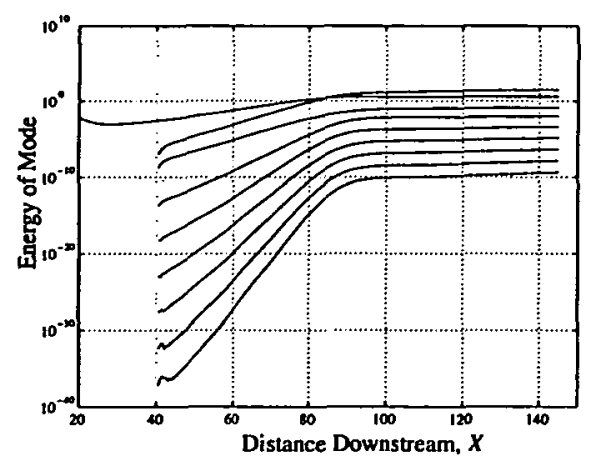

(a) $\mathbf{G}=0.2$

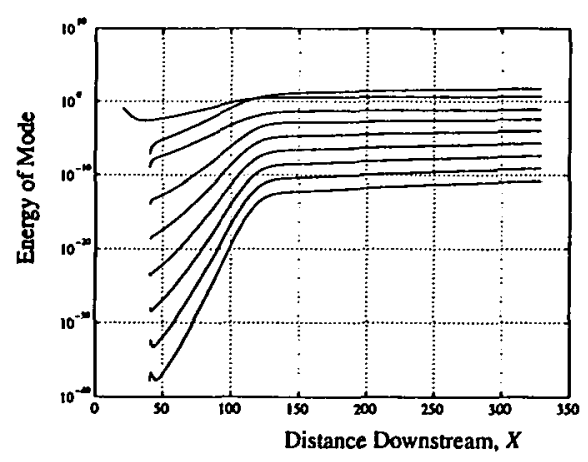

(b) $\mathbf{G}=0.05$

FIGURE 18. Energy of the first nine nonlinear modes in the unstably-stratified boundary layer, calculated for $G=0.2$ and $G=0.05$. The vortex wavenumber was 0.6 and $S=0$.

However, the temperature plot shows how the upwellings of fluid created by the 


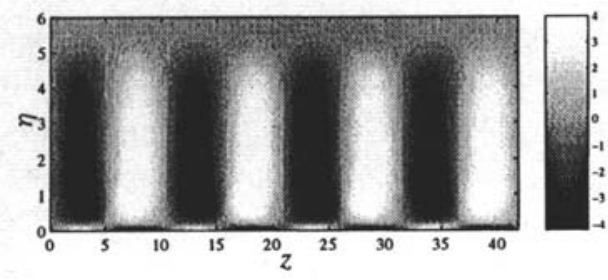

(a) Streamwise vorticity

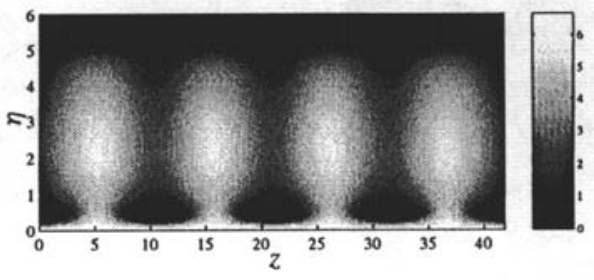

(b) Temperature

FIGURE 19. Contour plots of the streamwise vorticity and temperature associated with a Görtler number of 0.05 and a vortex wavenumber of 0.6 , just before code breakdown $(X \approx 320$ ) in the boundary layer. The basic-flow temperature profile was unstably stratified with $S=0$.

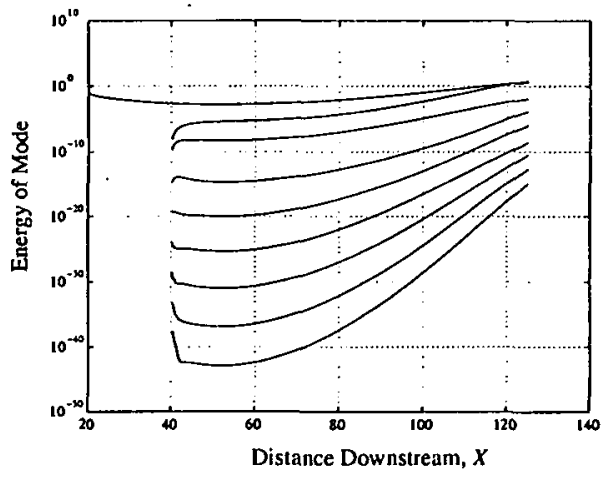

(a) $\mathrm{G}^{\mathrm{loc}}=1 / 2$

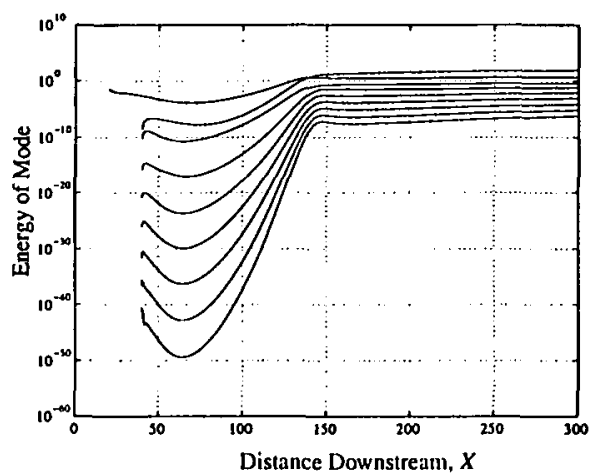

(b) $\mathrm{G}^{\mathrm{loc}}=-8$

FIGURE 20. Energy of the first nine nonlinear modes in the buoyancy-coupled boundary layer. The basic-flow temperature profile was stably stratified, the vortex wavenumber was 0.6 , the Görtler number 0.5 and $S=-1 / 2$. For (a) the streamwise velocity component of the basic flow is decelerated near the wall and conversely, it is accelerated for (b). In (b) the code was run to $X=300$ but no breakdown point was encountered in this period.

vortex pairs merge together, generating a band of fluid removed from the wall which is slower and warmer than the free stream. Since we are considering an unstable temperature stratification, "warmer" and "cooler" apply equally to both the nondimensional and the dimensional temperatures. If the flow was stably stratified, a non-dimensional temperature larger than the free stream would in fact mean that the dimensional temperature is smaller than the free stream (which would of course be warmer rather than cooler than the wall). Within this band there are still regions of extremely slow-moving, extra-hot fluid but these are gradually assimilated into the band itself as the system evolves downstream. Finally, below the band are concentrated jets of fluid which are accelerated to velocities beyond the free-stream value and which are much cooler than the surrounding fluid. 
5.2. Buoyancy coupling $(\mathbf{S} \neq \mathbf{0}$ ) As previously, we shall now consider how the introduction of buoyancy coupling in the basic flow equations alters the nonlinear solution. First of all the stably-stratified boundary layer will be considered. By setting a non-zero buoyancy parameter (in this case $-1 / 2$ ) and a positive value of $G^{\text {loc }}$ $(1 / 2$ here) the flow is decelerated in the streamwise direction near the wall, as depicted by Figure 1. Figure 20 (a) shows the energies of the first nine nonlinear modes in this case for a vortex wavenumber of 0.6 and a Görtler number of 0.5 . Just before code breakdown the basic-flow correction outgrows the fundamental mode, just as was found when no buoyancy coupling was present.

In comparison, Figure 20 (b) shows the energies of the same system but with $G^{\text {loc }}=-8$, therefore the streamwise velocity is accelerated beyond the free-stream value near the wall, creating a jet in the basic flow. In this case the code was run to $X=300$ without encountering a breakdown point; however, the basic-flow correction outgrows the fundamental mode much further upstream and after this point the mode energies increase only slightly.

As mentioned previously, these energy plots do not enable easy comparison between the stability of different cases, therefore in Figure 21 growth rates of the fundamental mode in each situation are shown. Also included is the growth rate of the fundamental mode when no buoyancy coupling is present, shown as a dashed-dotted line.

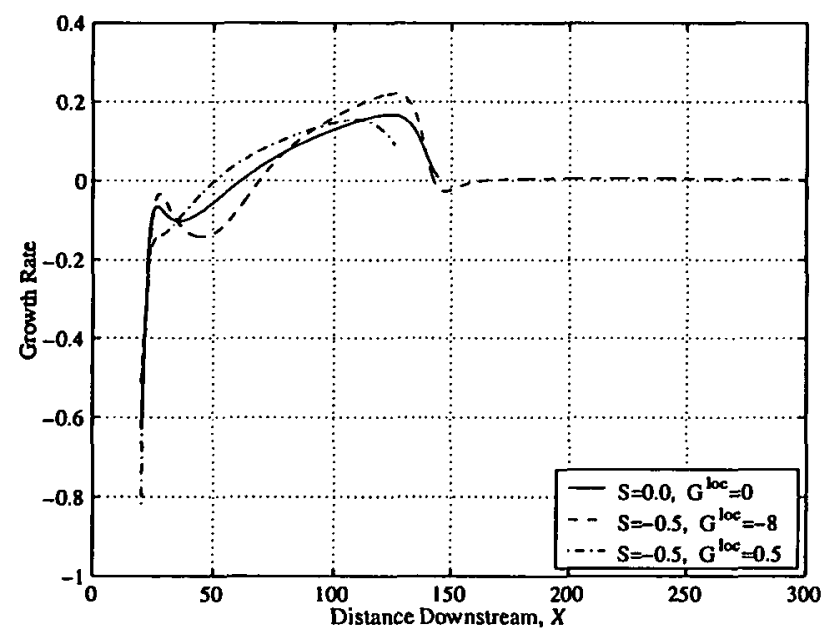

FIGURE 21. Growth rates of the fundamental mode for a stably-stratified basic flow and varying values of $S$ and $G^{\text {loc }}$. The vortex wavenumber was 0.6 and $G=0.5$.

This shows that the highest maximum growth rate occurs when buoyancy coupling is turned on and the basic flow is accelerated in the streamwise direction. The decelerated boundary layer and the boundary layer with no buoyancy coupling have similar maximum growth rates. However, it is the decelerated boundary layer which 


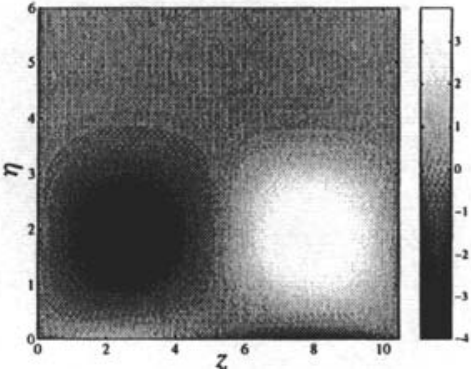

(a) Streamwise vorticity

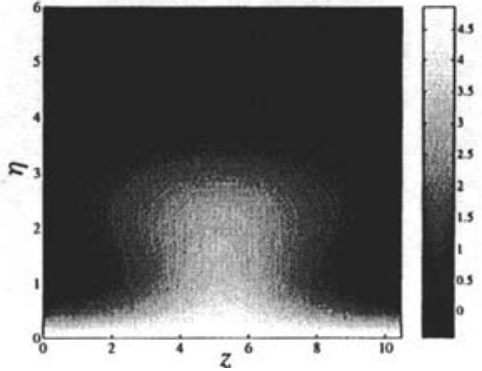

(b) Temperature

FIGURE 22. The total streamwise vorticity and temperature in the buoyancy-coupled, stably-stratified boundary layer $\left(S=-1 / 2, \mathrm{G}^{\text {loc }}=1 / 2\right)$ at $X=120$, just prior to code breakdown. The vortex wavenumber was 0.6 , the Görtler number 0.5 .

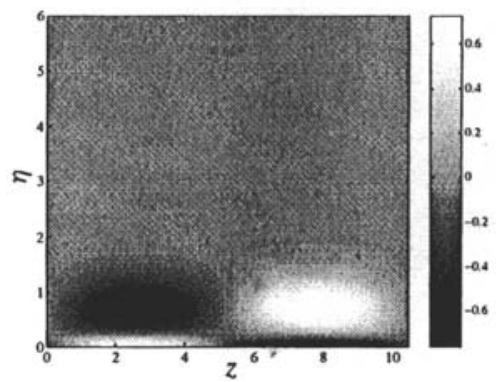

(a) Streamwise vorticity

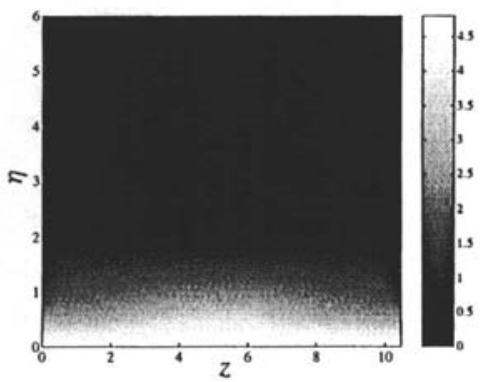

(b) Temperature

FIGURE 23. Contour plots of the streamwise vorticity and temperature, associated with a Görtler number of 0.5 and a vortex wavenumber of 0.6 , at the downstream location $X=120$ in the buoyancy-coupled boundary layer $\left(S=-1 / 2, G^{l o c}=-8\right)$. The basic-flow temperature profile was stably stratified.

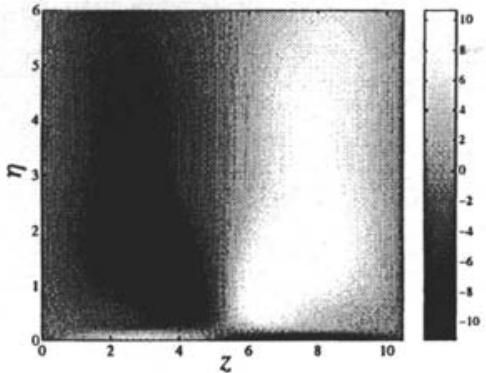

(a) Streamwise vorticity

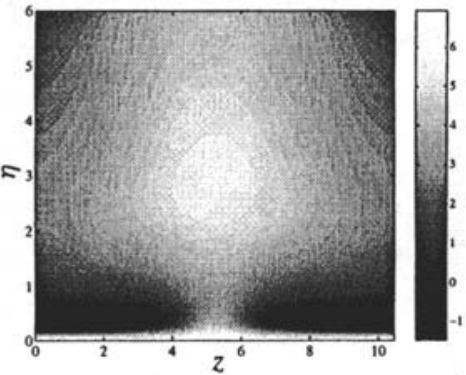

(b) Temperature

FIGURE 24. Contour plots of the streamwise vorticity and temperature, associated with a Görtler number of 0.5 and a vortex wavenumber of 0.6 , at the downstream location $X=300$ in the buoyancy-coupled boundary layer $\left(S=-1 / 2, G^{\text {loc }}=-8\right)$. The basic-flow temperature profile was stably stratified. 


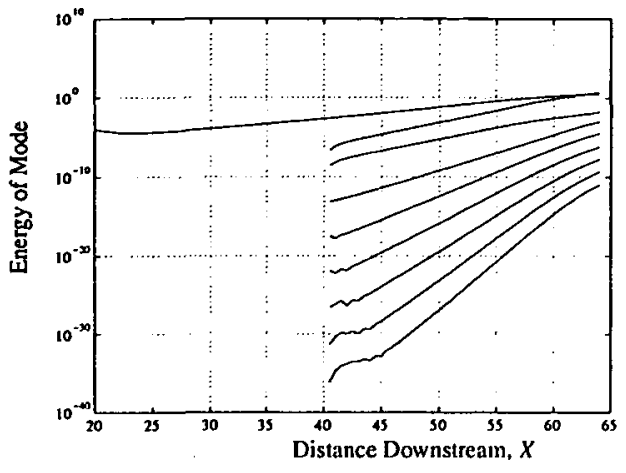

(a) $\mathrm{G}^{\text {loc }}=-3$

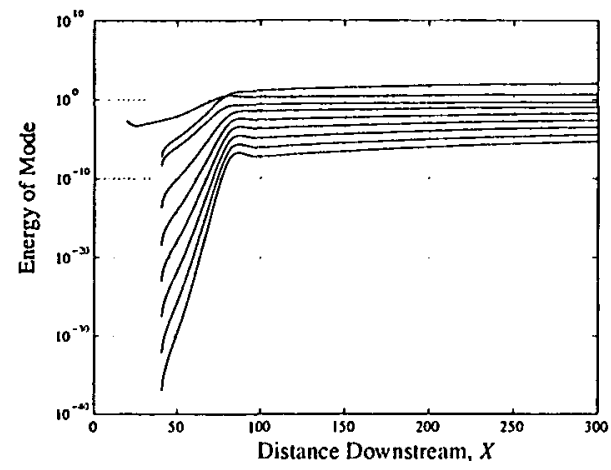

(b) $\mathrm{G}^{\text {loc }}=5$

FIGURE 25. Energy of the first nine nonlinear modes in the buoyancy-coupled boundary layer. The basicflow temperature profile was unstably stratified, the vortex wavenumber was 0.6 , the Görtler number 0.5 and $S=1$. For (a) the streamwise velocity component of the basic flow is decelerated near the wall and conversely it is accelerated in (b). In (b) the code was run to $X=300$ and no breakdown point was encountered in this period.

peaks first out of all three cases.

At this stage, parallels may be drawn with the linear solution given in Section 4.2. There it was found that, at small wavenumbers, accelerating the basic flow near the wall had the effect of decreasing the growth rate whilst decelerating it had the opposite effect. If the vortex wavenumber was increased so that the régime of the right-hand branch is entered, then the reverse becomes true-accelerating the flow in the streamwise direction increases the growth rate. As a relatively large wavenumber is being used here, it is the latter case which is applicable. Note, however, that the switching point from the linear to the nonlinear code is a very important factor (the linear solution is used as an initial condition for the nonlinear problem). The linear growth rates of Figure 5 show that in the initial stages the relative positions of the solutions for each value of $\mathrm{G}^{\mathrm{loc}}$ change twice before settling down to the situation described above. Therefore if the nonlinear code had been initiated during the small interval where accelerating the basic flow decreases the growth rate, our results would have been quite different.

In Figure 22 contour plots of the total streamwise vorticity and temperature are shown for the buoyancy-coupled, stably-stratified boundary layer with $S=-1 / 2$ and $\mathrm{G}^{\text {loc }}=1 / 2$. The values are shown for the downstream position $X=120$, just prior to code breakdown. These should be compared with Figures 23 which show the same quantities for the accelerated case $\left(\mathrm{G}^{\mathrm{loc}}=-8\right)$, again at $X=120$. Both cases correspond to a vortex wavenumber of 0.6. A major difference between the two sets of figures is the extent of the vortices. In the former (decelerated) case, the vortices are large and extend a long way from the wall. In the latter (accelerated) case, the 


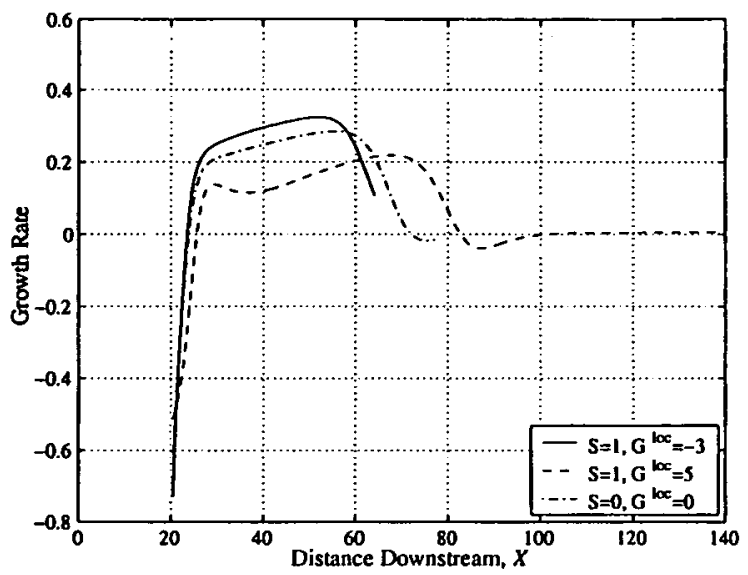

FIGURE 26. Growth rates of the fundamental modes of the unstably-stratified boundary layer, with $S=1$, $\mathrm{G}^{\mathrm{loc}}=-3 ; \mathrm{S}=1, \mathrm{G}^{\mathrm{loc}}=5$ and $\mathrm{S}=\mathrm{G}^{\mathrm{loc}}=0$. The vortex wavenumber was 0.6 , the Görtler number 0.5 .

vortices are much more compact and are positioned closer to the wall. It is not only the vortices which are affected however; in Figure 23 (a) the jet of accelerated fluid can clearly be seen. This appears to inhibit the movement of the fluid away from the wall, since only a small upwelling is visible by $X=120$ but when $G^{\text {loc }}=1 / 2$ and the basic flow is decelerated the mushroom shape is already apparent and the code is close to breakdown. As the disturbance develops (Figure 24) the faster-moving fluid from the jet remains in two localised pools either side of the column of fluid pulled up by the vortices. Normally the fluid in the head of this column would be much slower than the surrounding flow (in the same way that it is much hotter, as shown in Figure 17 (b)); however, the basic-flow jet seems to dilute this effect and the velocity disturbance spreads out to either side, joining with the disturbance in the adjacent wavelength period (Figure 24 (b)).

Similar effects are found when the basic flow is unstably stratified. Energy plots are shown in Figures 25 (a) and 25 (b) for a decelerated $\left(G^{\text {loc }}=-3\right)$ and an accelerated $\left(G^{\text {loc }}=5\right)$ basic flow respectively. In each case the Görtler number was 0.5 and the vortex wavenumber 0.6 .

When the basic flow is decelerated, the code breaks down soon after the basicflow correction outgrows the fundamental mode. When it is accelerated beyond the freestream value, the code can be run to $X=300$ without encountering breakdown and for much of this time the basic-flow correction has a larger energy than the fundamental mode.

Once again, to enable comparison between the two cases, growth rates of the fundamental modes are plotted in Figure 26 along with the growth rate when no buoyancy coupling is present. This time, with the unstable stratification, the most 


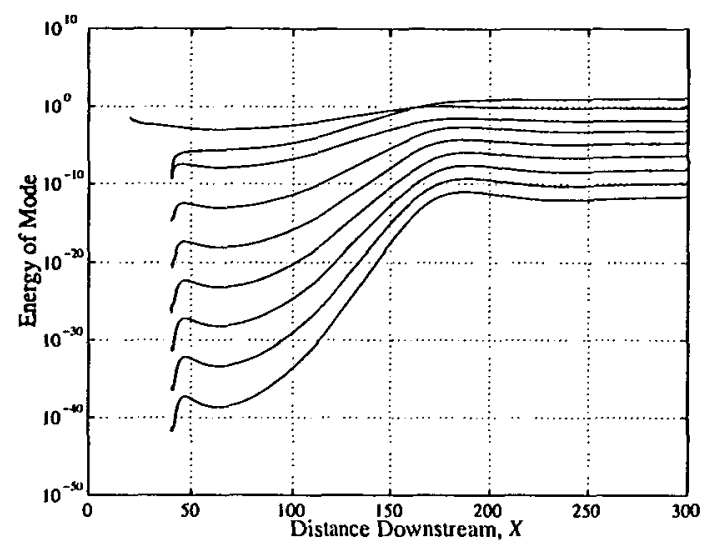

FIGURE 27. Energy of the first nine nonlinear modes in the stably-stratified boundary layer. The Görtler number was $-0.5, \mathrm{~S}=-1 / 2, \mathrm{G}^{\mathrm{loc}}=-16$ and the vortex wavenumber was 0.6 . Code breakdown is not encountered in the section shown.

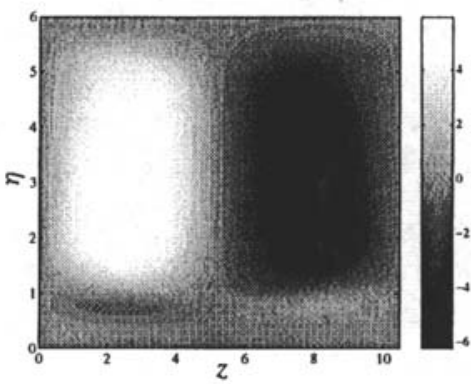

(a) Streamwise vorticity

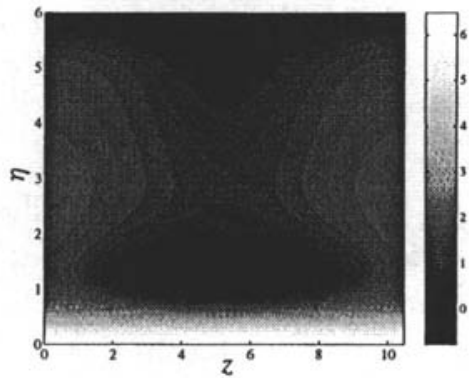

(b) Temperature

FIGURE 28. The total streamwise vorticity and the temperature for a stably-stratified boundary layer. The Görtler number was $-0.5, S=-1 / 2, G^{\text {loc }}=-16$ and the vortex wavenumber was 0.6 . These values are for the downstream location $X=300$.

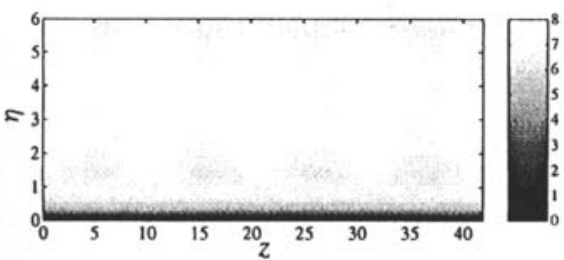

(a) Streamwise vorticity

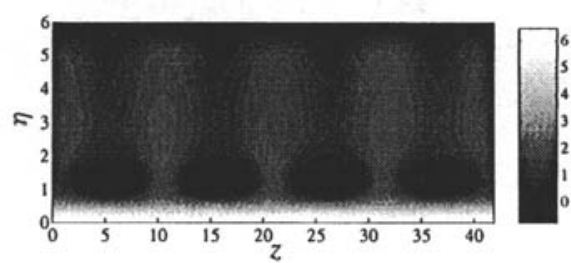

(b) Temperature

FIGURE 29. The total streamwise velocity and the temperature for a stably-stratified boundary layer. The Görtler number was $-0.5, \mathrm{~S}=-1 / 2, \mathrm{G}^{\text {loc }}=-16$ and the vortex wavenumber was 0.6 . These values are for the downstream location $X=300$. 
unstable situation occurs when the basic flow velocity is decelerated in the streamwise direction. This again ties in with the inviscid results of Stott and Denier [23] and also the linear results of Section 4 . These linear results showed that a larger vortex wavenumber is required in the unstably-stratified case than in the stably-stratified case to reverse the situation.

Finally, we return to the situation first discussed in Section 4.3, that is, the destabilisation of the stably-stratified boundary layer over a wall with convex curvature. It was discovered that this otherwise stable situation could be destabilised with the addition of buoyancy coupling in the basic-flow equations, such that the streamwise velocity component is accelerated beyond the free stream value. In Figure 27, the energy of the first nine nonlinear modes is shown for such a boundary layer. In this case the Görtler number was $-0.5, S=-1 / 2$ and $G^{\text {loc }}=-16$. The vortex wavenumber was again 0.6. This plot is very similar to the other energy plots which have been discussed in this section; that is, after a certain distance downstream the energy of basic-flow correction becomes larger than that of the fundamental mode. In this case, as with the other cases we have considered where a jet has been present in the basic flow velocity, code breakdown is not achieved in the downstream section examined. In Figures 28 contour plots of the total streamwise vorticity and total temperature respectively are given for the downstream location $X=300$.

The first of this set of figures, showing the vorticity, is immediately obviously different to all other vorticity plots presented in this section as the vortices have been shifted in the spanwise direction by half a wavelength. Thus the pair shown in the contour plots act to pull fluid down towards the wall rather than away from it. A second notable difference is that fluid only moves from the area at the top edge of the boundary layer. This means that the slower moving, warmer fluid nearest the wall is unmoved from its original position. Usually it would be this fluid that is drawn up into the head of the mushroom-shaped upwellings (see for example Figure 14), but in this case it is fluid from the very top of the boundary layer which ends up in the heads; that is, the super-fast fluid which originally formed the jet in the basic flow.

Therefore, as shown by Figure 29, the vortices act to construct circular regions in the flow which contain fluid moving much faster than the free stream. Unlike previous cases where the curvature was concave, there is little difference between the temperature of these jets and the surrounding fluid.

\section{Conclusions}

In Section 4 the linear problem was solved. We began by considering the Hiemenz boundary layer, disabling buoyancy coupling in the basic flow. This problem is already reasonably well understood and only a few results were presented. These nonetheless 
demonstrated that the combined Grashof-Görtler situation is more complicated than either singular problem, due to the presence of transient growth. This is manifested within the boundary layer in the same way as it has been found to reside within the mixing layer [25]; it is seen in the form of oscillatory growth rates and folded neutral curves. Analysis of the inviscid stability boundaries showed that the critical Görtler number is proportional to $X^{-1 / 3}$ for a stably-stratified basic flow and $-X^{-1 / 3}$ for an unstably-stratified basic flow. Thus the degree of curvature required for instability changes with the downstream coordinate and periods of transient growth may be followed by sustained growth (equally periods of sustained growth may be followed by decay).

Transient growth persists with the addition of buoyancy coupling, the degree of which is controlled via the buoyancy parameter, $\mathrm{S}$. By varying the value of $\mathrm{G}^{\text {loc }}$ (which describes the local gradient of the wall), it is possible to accelerate or decelerate the streamwise velocity component of the basic flow near the wall. In agreement with the findings of Stott and Denier [23] for the inviscid problem, it was found that accelerating the flow in this manner at small vortex wavenumbers (of the order $10^{-1}$ ) decreased the growth rate whilst decelerating it had the opposite effect. However, further to this it was found that at large wavenumbers (the right-hand branch régime) the situation was reversed; accelerating the basic flow near the wall increases the growth rate whilst decelerating it decreases the growth rate as the extra energy is removed from the boundary layer. It appears that in the small wavenumber limit this extra energy is put into transient rather than sustained downstream growth. The reason for this is not entirely clear, compounded by the fact that the inviscid stability boundaries are not immediately obvious for the buoyancy-coupled boundary layer, and perhaps further work is called for on the matter.

An analytical expression was given for the asymptote to the right-hand branch in the local wavenumber-local Görtler number plane in Section 4.3. It was found that there are in fact two right-hand branch asymptotes which apply to the buoyancy-coupled boundary layer, one with a positive gradient and one with a negative gradient. This latter branch applies to neutral curves calculated for convex curvatures and exists even when the basic-flow temperature profile has a stable temperature stratification; an otherwise completely stable situation. The gradient of this asymptote tends swiftly to minus infinity as $\mathrm{G}^{\mathrm{loc}}$ becomes increasingly negative, thus it is only in flows which have an accelerated basic flow in the streamwise direction that the effects will be seen. Therefore in addition to curvature and stratification we now have a third factor which may render the boundary layer unstable to longitudinal vortex motions.

Section 5 dealt with the solution to the corresponding nonlinear problem. The system was solved using essentially the same method as for the linear problem; a finite-difference scheme in the normal direction coupled with a Crank-Nicolson marching scheme in the downstream direction. 
Initially the Hiemenz basic flow was considered, as given by the solution of the equations (2.2) with the buoyancy parameter, $S$, set to zero. Investigations showed that at small values of the vortex wavenumber the basic-flow correction was outgrown rapidly by the second mode - however, the energy of this did not supersede that of the fundamental mode before code breakdown was reached. If the wavenumber is then increased by an order of magnitude a more familiar situation is found. That is, the basic-flow correction remains larger than the second mode throughout and indeed outgrows the fundamental mode shortly before breakdown. The cause of this breakdown is surmised to be the result of reverse flow thus violating the parabolic governing equations. Another feature of the solution at the larger wavenumber is that the vortices and the resultant upwellings of fluid are spread across the space of an entire wavelength, whereas at the smaller wavenumber they are concentrated toward the centre of the wavelength.

As would of course be expected, it was found that the most unstable scenario is an unstable temperature stratification with a concave wall curvature. The greater the degree of either mechanism, the more unstable the system becomes.

Next the buoyancy-coupled basic flow was considered. As with both the inviscid [23] and the linear solutions, it was found that decelerating or accelerating the basic flow had a large effect on the growth of the disturbance. However, the effect also depends upon the magnitude of the vortex wavenumber.

The presence of the jet over a concavely curving wall has a diluting effect upon the fluid in the head of the mushroom-shaped upwelling. With no jet present, the fluid is this region is much slower than the surrounding fluid - in fact it ultimately stops flowing in the downstream direction at all. This, as stated many times previously, is the cause of code breakdown. However, with the jet in the basic flow the fluid is not allowed to slow so drastically and thus code breakdown was not observed over the downstream stretch considered here.

Finally, in the course of the linear studies it was discovered that this jet may, by itself, destabilise an otherwise stable situation. This is found also to be true of the nonlinear solution. It is found that the vortices are shifted by half a wavelength in the spanwise direction and that they move fluid only from the outer edge of the boundary layer rather than taking it from the wall region. Thus the heads of the fluid upwellings are filled with accelerated rather than virtually stationary fluid and they do not differ greatly in temperature from the surrounding environment. It would be interesting to see if the same mechanism could be used in the mixing layer to destabilise the system; and indeed whether such results may be reproduced in the laboratory. We also note that the presence of the jet implies the propensity of the situation to be unstable to inviscid travelling waves. In addition the presence of the vortices will may lead to additional secondary instabilities (Cole, Otto and Watson [4]). 


\section{Acknowledgements}

CEW would like to thank both the EPSRC for providing funding for this research and Dr. Terry Cole for allowing use and modification of the basic nonlinear code.

\section{References}

[1] A. Benmalek and W. S. Saric, "Effects of curvature variations on the nonlinear evolution of Goertler vortices", Phys. Fluids 6 (1994) 3353-3367.

[2] H. Blasius, "Grenzschichten in flüssigkeiten mit kleiner Reibung", Z. Math. Phys. 56 (1908) 1-37.

[3] T. Cebeci and P. Bradshaw, Momentum transfer in boundary layers (Hemisphere, London, 1977).

[4] T. R. Cole, S. R. Otto and C. E. Watson, "The nonlinear growth of Görtler vortices in curved mixing layers and their effect on the inherent inviscid modes", 2005, (in preparation).

[5] J. P. Denier and P. Hall, "On the nonlinear development of the most unstable Görtler vortex mode", J. Fluid Mech. 247 (1993) 1-16.

[6] J. P. Denier, P. Hall and S. O. Seddougui, "On the receptivity problem for Görtler vortices: vortex motions induced by wall roughness", Philos. Trans. Roy. Soc. London A 335 (1991) 51-85.

[7] H. Görtler, "On the three-dimensional instability of laminar boundary layers on concave walls", NACA Tech. Mem. (1940) 1375.

[8] P. Hall, "Taylor-Görtler vortices in fully developed or boundary layer flows: linear theory", $J$. Fluid Mech. 124 (1982) 475-494.

[9] P. Hall, "The linear development of Görtler vortices in growing boundary layers", J. Fluid Mech. 130 (1983) 41-58.

[10] P. Hall, "The nonlinear development of Görtler vortices in growing boundary layers", J. Fluid Mech. 193 (1988) 243-266.

[11] P. Hall, "Görtler vortices in growing boundary layers: the leading edge receptivity problem, linear growth and the nonlinear breakdown stage", Mathematika 37 (74) (1990) 151-189.

[12] P. Hall, "Streamwise vortices in heated boundary layers", J. Fluid Mech. 252 (1993) 301-324.

[13] P. Hall and W. D. Lakin, "The fully nonlinear development of Görtler vortices in growing boundary layers", Proc. Roy. Soc. London A 415 (1988) $421-444$.

[14] P. Hall and H. Morris, "On the instability of boundary layers on heated flat plates", J. Fluid Mech. 245 (1992) 367-400.

[15] N. Itoh, "A non-parallel theory for Görtler instability of Falkner-Skan boundary layers", Fluid Dyn. Res. 28 (2001) 383-396.

[16] K. Lee and J. T. C. Liu, "On the growth of mushroomlike structures in nonlinear spatially developing Goertler vortex flow", Phys. Fluids A 4 (1992) 95-103.

[17] S. M. Mangalam, J. R. Dagenhart, T. E. Hepner and J. F. Meyers, "The Görtler instability on an airfoil", 23rd Aerodynamic Sciences Meeting, January 14-17, 1985, AIAA-85-049I.

[18] S. R. Otto, J. A. K. Stott and J. P. Denier, "On the role of buoyancy in determining the stability of curved mixing layers", Phys. Fluids 11 (6) (1999) 1495-1501.

[19] D. J. Owen, S. O. Seddougui and S. R. Otto, "The linear evolution of centrifugal instabilities in curved, compressible mixing layers", Phys. Fluids 9 (1997) 2506-2518.

[20] J. M. Sarkies and S. R. Otto, "Görtler vortices in compressible mixing layers", J. Fluid Mech. 427 (2001) 359-388.

[21] R. S. Scorer, Dynamics of meteorology and climate, Wiley Praxis Series in Atmospheric Physics (John Wiley \& Sons, Chichester, 1997). 
[22] E. A. Spiegel and G. Veronis, "On the Boussinesq approximation for a compressible fluid", $J$. Astrophys. 131 (1960) 442-447.

[23] J. A. K. Stott and J. P. Denier, "The stability of boundary layers on curved heated plates", ANZIAM J. 43 (2002) 333-358.

[24] J. D. Swearingen and R. F. Blackwelder, "The growth and breakdown of streamwise vortices in the presence of a wall", J. Fluid Mech. 182 (1987) 255.

[25] C. E. Watson, S. R. Otto and T. L. Jackson, "The receptivity and evolution of longitudinal vortices: the interplay between buoyancy and curvature within mixing layers", 2005, submitted to Theor. Comp. Fluid Dynamics.

[26] R. S. Wu and K. C. Cheng, "Thermal instability of Blasius flow along horizontal plates", Intl. J. Heat Mass Transfer 105 (1976) 907-913. 